\title{
Non-linear Evolution of Baryon Acoustic Oscillations from Improved Perturbation Theory in Real and Redshift Spaces
}

\author{
Atsushi Taruya ${ }^{1,2}$, Takahiro Nishimichi ${ }^{3}$, Shun Saito ${ }^{3}$, Takashi Hiramatsu ${ }^{4}$ \\ ${ }^{1}$ Research Center for the Early Universe, School of Science, \\ University of Tokyo, Bunkyo-ku, Tokyo 113-0033, Japan \\ ${ }^{2}$ Institute for the Physics and Mathematics of the Universe, \\ University of Tokyo, Kashiwa, Chiba 277-8568, Japan \\ ${ }^{3}$ Department of Physics, University of Tokyo, 113-0033, Japan and \\ ${ }^{4}$ Institute for Cosmic Ray Research, University of Tokyo, Kashiwa, Chiba 277-8582, Japan
}

(Dated: October 26, 2018)

\begin{abstract}
We study the non-linear evolution of baryon acoustic oscillations in the matter power spectrum and correlation function from the improved perturbation theory (PT). Based on the framework of renormalized PT, which provides a non-perturbative way to treat the gravitational clustering of large-scale structure, we apply the closure approximation that truncates the infinite series of loop contributions at one-loop order, and obtain a closed set of integral equations for power spectrum and non-linear propagator. The resultant integral expressions are basically equivalent to those previously derived in the form of evolution equations, and they keep important non-perturbative properties which can dramatically improve the prediction of non-linear power spectrum. Employing the Born approximation, we then derive the analytic expressions for non-linear power spectrum and the predictions are made for non-linear evolution of baryon acoustic oscillations in power spectrum and correlation function. We find that the improved PT possesses a better convergence property compared with standard PT calculation. A detailed comparison between improved PT results and N-body simulations shows that a percent-level agreement is achieved in a certain range in power spectrum and in a rather wider range in correlation function. Combining a model of nonlinear redshift-space distortion, we also evaluate the power spectrum and correlation function in redshift space. In contrast to the results in real space, the agreement between N-body simulations and improved PT predictions tends to be worse, and a more elaborate modeling for redshift-space distortion needs to be developed. Nevertheless, with currently existing model, we find that the prediction of correlation function has a sufficient accuracy compared with the cosmic-variance errors for future galaxy surveys with volume of a few $h^{-3} \mathrm{Gpc}^{3}$ at $z \gtrsim 0.5$.
\end{abstract}

PACS numbers: 98.80.-k

\section{INTRODUCTION}

In the last decade, systematic measurements of the cosmic microwave background anisotropies as well as large-scale structure of the Universe have led to the establishment of the "standard cosmological model" (e.g., [1, 2, 13 , 4, , 5]). The Universe is close to a flat geometry, and is filled with the hypothetical cold dark matter (CDM) particles, together with a small fraction of baryons, which serve as the seeds of structure formation of the Universe. The most striking feature in the standard cosmological model is that the energy contents of the Universe is dominated by the mysterious energy component called dark energy, which is supposed to drive the late-time cosmic acceleration discovered by the observation of distant supernovae (e.g., [6, 7])).

Currently, our understanding of the nature of dark energy is still lacking. Although the observation is roughly consistent with cosmological constant and with no evidence for time dependence of dark energy, long-distance modifications of general relativity have been proposed alternative to the dark energy and these reconcile with the observation of late-time acceleration (see 8, 9, 10, 11] for reviews). While a fully consistent model of modified gravity has not yet been constructed ( see [12, 13, 14] for popular models), a possibility of break-down of general relativity still remains and should be tested.

To understand deeply the nature of dark energy or origin of cosmic acceleration, a further observational study is definitely important. There are two comprehensive ways to distinguish between many models of dark energy and discriminate the dark energy from modified gravity. One is to precisely measure the expansion history of the Universe, and the other is to observe the growth of structure.

Among various observational techniques, baryon acoustic oscillations (BAOs) imprinted on the matter power spectrum or two-point correlation function can be used as a standard ruler to measure the cosmic expansion history (e.g., 
[15, 16], see also [17, 18, 19, 20, 21] for recent BAO measurements). The characteristic scale of BAOs, which is determined by the sound horizon scale of primeval baryon-photon fluid at the last scattering surface [22, 23], is thought to be a robust measure and it lies on the linear to quasi-linear regimes of the gravitational clustering of large-scale structure [24, 25]. With a percent-level determination of the characteristic scale of BAOs, the expansion history can be tightly constrained, and the equation-of-state parameter of the dark energy, $w_{\text {de }}$, defined by the ratio of pressure to energy density of dark energy, would be precisely determined within the precision of a few $\%$ level [26, 27]. This is the basic reason why most of the planned and ongoing galaxy redshift surveys aim at precisely measuring the BAOs (e.g., [28, 29, 30, 31]).

While the robustness of the BAOs as a standard ruler has been repeatedly stated and emphasized in the literature, in order to pursue an order-of-magnitude improvement, a precise theoretical modeling of BAOs definitely plays an essential role for precision measurement of $\mathrm{BAO}$ scale, and it needs to be investigated taking account of the various systematic effects. Among these, the non-linear clustering and redshift-space distortion effects as well as the galaxy biasing cannot be neglected, and affect the characteristic scale, although their effects are basically moderate at the relevant wavenumber, $k \lesssim 0.3 h \mathrm{Mpc}^{-1}$.

Recently, several analytic approaches to deal with the non-linear clustering have been developed, complementary to the N-body simulations $32,33,34,35,36,37,38,39,40,41,42]$. In contrast to the standard analytical calculation with perturbation theory (PT), these have been formulated in a non-perturbative way with techniques resumming a class of infinite series of higher-order corrections in perturbative calculation. Thanks to its non-perturbative formulation, the applicable range of the prediction is expected to be greatly improved, and the non-linear evolution of baryon acoustic oscillations would be accurately described with a percent-level precision.

The purpose of this paper is to investigate the viability of this analytic approach, focusing on a specific improved treatment. In the previous paper [43], we have applied a non-linear statistical method, which is widely accepted in the statistical theory of turbulence [44], to the cosmological perturbation theory of large-scale structure. We have derived the non-perturbative expressions for the power spectrum, coupled with non-linear propagator, which effectively contain the information on the infinite series of higher-order corrections in the standard PT expansion. Based on this formalism, the analytic treatment of the non-perturbative expression is developed employing the Born approximation, and the leading-order calculation of power spectrum is compared with N-body simulations in real space [45], finding that a percent-level agreement is achieved in a mildly non-linear regime (see also [46]). Here, we extend the analysis to those including the next-to-leading order corrections of Born approximation. In addition to the power spectrum, we will consider the two-point correlation function, paying a special attention on the baryon acoustic peak, i.e., a Fourier counterpart of BAOs in power spectrum. Further, we also discuss the non-linear clustering in redshift space, and the predictions of improved PT are compared with N-body results, combining a non-linear model of redshift-space distortion. We examine how well the present non-linear model accurately describe the systematic effects on BAOs and/or baryon acoustic peak.

This paper is organized as follows. In Sec. II] we briefly mention the basic equations for cosmological PT as our fundamental basis to deal with the non-linear gravitational clustering. We then discuss in some details in Sec. III how to compute the non-linear power spectrum or two-point correlation functions. Starting from the discussions on standard treatment of perturbative calculation and its non-perturbative reformulation called renormalized PT, we introduce the closure approximation, which gives a consistent non-perturbative scheme to treat the infinite series of renormalized PT expansions, and obtain a closed set of non-perturbative expressions for power spectrum. Based on this, we present a perturbative treatment of the closed set of equations while keeping important non-perturbative properties. Section IV gives the main result of this paper, in which a detailed comparison between improved PT calculation and N-body simulation is made, especially focusing on the non-linear evolution of BAOs. We compute the power spectrum and two-point correlation function in both real and redshift spaces, and investigate the accuracy of both predictions by comparing improved PT with N-body results. Finally, section $\mathrm{V}$ is devoted to the discussion and conclusion.

\section{PRELIMINARIES}

Throughout the paper, we consider the evolution of cold dark matter (CDM) plus baryon systems neglecting the tiny fraction of (massive) neutrinos. Owing to the single-stream approximation of the collisionless Boltzmann equation, which is thought to be a quite accurate approximation on large scales, the evolution of the CDM plus baryon system can be treated as the irrotational and pressureless fluid system whose governing equations are continuity and Euler equations in addition to the Poisson equation (see Ref. [47] for review). In the Fourier representation, these equations are further reduced to a more compact form. Let us introduce the two-component vector (e.g., 32] ):

$$
\Phi_{a}(\boldsymbol{k} ; t)=\left(\delta(\boldsymbol{k} ; t),-\frac{\theta(\boldsymbol{k} ; t)}{f(t)}\right),
$$


where the subscript $a=1,2$ selects the density and the velocity components of CDM plus baryons, with $\delta$ and $\theta(\boldsymbol{x}) \equiv \nabla \cdot \boldsymbol{v}(\boldsymbol{x}) /(a H)$, where $a$ and $H$ are the scale factor of the Universe and the Hubble parameter, respectively. The function $f(t)$ is given by $f(t) \equiv d \ln D(t) / d \ln a$ and the quantity $D(t)$ being the linear growth factor. Then, in terms of the new time variable $\eta \equiv \ln D(t)$, the evolution equation for the vector quantity $\Phi_{a}(\boldsymbol{k} ; t)$ becomes

$$
\left[\delta_{a b} \frac{\partial}{\partial \eta}+\Omega_{a b}(\eta)\right] \Phi_{b}(\boldsymbol{k} ; \eta)=\int \frac{d^{3} \boldsymbol{k}_{1} d^{3} \boldsymbol{k}_{2}}{(2 \pi)^{3}} \delta_{D}\left(\boldsymbol{k}-\boldsymbol{k}_{1}-\boldsymbol{k}_{2}\right) \gamma_{a b c}\left(\boldsymbol{k}_{1}, \boldsymbol{k}_{2}\right) \Phi_{b}\left(\boldsymbol{k}_{1} ; \eta\right) \Phi_{c}\left(\boldsymbol{k}_{2} ; \eta\right),
$$

where $\delta_{D}$ is the Dirac delta function. Here and in what follows, we use the summation convention that the repetition of the same subscripts indicates the sum over the whole vector components. The time-dependent matrix $\Omega_{a b}(\eta)$ is given by

$$
\Omega_{a b}(\eta)=\left(\begin{array}{cc}
0 & -1 \\
-\frac{3}{2 f^{2}} \Omega_{\mathrm{m}}(\eta) & \frac{3}{2 f^{2}} \Omega_{\mathrm{m}}(\eta)-1
\end{array}\right)
$$

The quantity $\Omega_{\mathrm{m}}(\eta)$ is the density parameter of CDM plus baryons at a given time. Each component of the vertex function $\gamma_{a b c}$ becomes

$$
\gamma_{a b c}\left(\boldsymbol{k}_{1}, \boldsymbol{k}_{2}\right)=\left\{\begin{array}{cl}
\frac{1}{2}\left\{1+\frac{\boldsymbol{k}_{2} \cdot \boldsymbol{k}_{1}}{\left|\boldsymbol{k}_{2}\right|^{2}}\right\} & ;(a, b, c)=(1,1,2) \\
\frac{1}{2}\left\{1+\frac{\boldsymbol{k}_{1} \cdot \boldsymbol{k}_{2}}{\left|\boldsymbol{k}_{1}\right|^{2}}\right\} & ;(a, b, c)=(1,2,1) \\
\frac{\left(\boldsymbol{k}_{1} \cdot \boldsymbol{k}_{2}\right)\left|\boldsymbol{k}_{1}+\boldsymbol{k}_{2}\right|^{2}}{2\left|\boldsymbol{k}_{1}\right|^{2}\left|\boldsymbol{k}_{2}\right|^{2}} & ;(a, b, c)=(2,2,2) \\
0 & ; \text { otherwise }
\end{array} .\right.
$$

Note that the formal solution of $\Phi_{a}$ can be obtained from Eq. (2.2) and is expressed as (e.g., [32, 47])

$$
\Phi_{a}(\boldsymbol{k} ; \eta)=g_{a b}\left(\eta, \eta_{0}\right) u_{b} \delta_{0}(\boldsymbol{k})+\int_{\eta_{0}}^{\eta} d \eta^{\prime} g_{a b}\left(\eta, \eta^{\prime}\right) \int \frac{d^{3} \boldsymbol{k}_{1} d^{3} \boldsymbol{k}_{2}}{(2 \pi)^{3}} \delta_{D}\left(\boldsymbol{k}-\boldsymbol{k}_{1}-\boldsymbol{k}_{2}\right) \gamma_{b c d}\left(\boldsymbol{k}_{1}, \boldsymbol{k}_{2}\right) \Phi_{c}\left(\boldsymbol{k}_{1} ; \eta^{\prime}\right) \Phi_{d}\left(\boldsymbol{k}_{2} ; \eta^{\prime}\right)
$$

Here, the quantity $u_{a}$ is the constant vector which specifies the initial condition (see next section), and the quantity $g_{a b}$ denotes the linear propagator satisfying the following equation:

$$
\left[\delta_{a b} \frac{\partial}{\partial \eta}+\Omega_{a b}(\eta)\right] g_{b c}\left(\eta, \eta^{\prime}\right)=0
$$

with the boundary condition $g_{a b}(\eta, \eta)=\delta_{a b}$. The quantity $\delta_{0}$ is the random density field given at an early time $\eta_{0}$, which is assumed to obey the Gaussian statistic. The power spectrum of density field is defined as

$$
\left\langle\delta_{0}(\boldsymbol{k}) \delta_{0}\left(\boldsymbol{k}^{\prime}\right)\right\rangle=(2 \pi)^{3} \delta_{D}\left(\boldsymbol{k}+\boldsymbol{k}^{\prime}\right) P_{0}(k) .
$$

Eq. (2.2) or (2.5) is the fundamental building block of large-scale structure, and the three quantities $\gamma_{a b c}, g_{a b}$ and $P_{0} u_{a} u_{b}$ introduced here constitute the basic pieces of standard PT. The graphical representation of them is shown in Fig. 1] (see also Ref. [32]).

\section{IMPROVED PERTURBATION THEORY}

\section{A. Standard PT vs. Renormalized PT}

In this paper, we are especially concerned with the non-linear evolution of the two-point statistics, defined as the ensemble average of $\Phi_{a}$ :

$$
\left\langle\Phi_{a}(\boldsymbol{k} ; \eta) \Phi_{b}\left(\boldsymbol{k}^{\prime} ; \eta^{\prime}\right)\right\rangle=(2 \pi)^{3} \delta_{\mathrm{D}}\left(\boldsymbol{k}+\boldsymbol{k}^{\prime}\right) P_{a b}\left(|\boldsymbol{k}| ; \eta, \eta^{\prime}\right) ; \quad \eta \geq \eta^{\prime}
$$

In the above, there are four types of power spectra, $P_{11}, P_{12}, P_{21}$ and $P_{22}$, which respectively correspond to the autoand cross-power spectra, $P_{\delta \delta},-P_{\delta \theta} / f,-P_{\theta \delta} / f$ and $P_{\theta \theta} / f^{2}$. Note that in general we have $P_{12} \neq P_{21}$ unless $\eta=\eta^{\prime}$. 


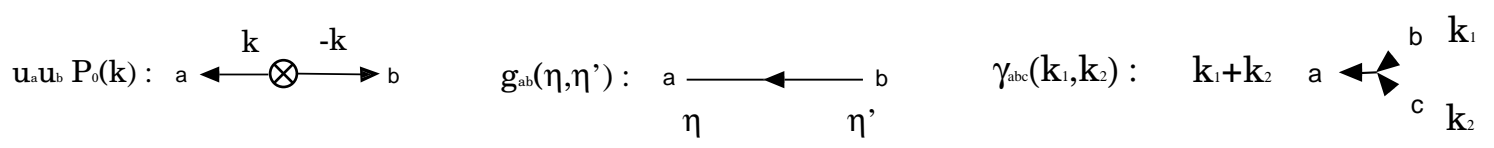

FIG. 1: Diagrammatic notion of the initial power spectrum (left), linear propagator (middle), and tree vertex (right). The linear propagator satisfies the equation (2.6) with boundary condition $g_{a b}(\eta, \eta)=\delta_{a b}$. The explicit expression of vertex function $\gamma_{a b c}$ is given by Eq. (2.4).

Consider how to compute the power spectrum based on the analytic treatment. In the standard treatment of the perturbation theory, we first assume that the field $\Phi_{a}$ is a small perturbed quantity and it is expanded as

$$
\Phi_{a}(\boldsymbol{k} ; \eta)=\Phi_{a}^{(1)}(\boldsymbol{k} ; \eta)+\Phi_{a}^{(2)}(\boldsymbol{k} ; \eta)+\Phi_{a}^{(3)}(\boldsymbol{k} ; \eta)+\cdots
$$

The explicit functional form of the quantity $\Phi_{a}^{(n)}$ is systematically derived through the order-by-order treatment of Eq. (2.2). Substituting the above expansion into the definition (3.1) and evaluating it perturbatively, the power spectrum $P_{a b}(k ; \eta, \eta)$, shortly abbreviated as $P_{a b}(k ; \eta)$, is schematically expressed as

$$
P_{a b}(k ; \eta)=e^{2 \eta} u_{a} u_{b} P_{0}(k)+P_{a b}^{1-\text { loop }}(k ; \eta)+P_{a b}^{2-\text { loop }}(k ; \eta)+\cdots
$$

where we chose $u_{a}=(1,1)$, which implies that the growing-mode solution is imposed at the initial condition ${ }^{1}$. The function $P_{0}(k)$ is the linear power spectrum given at an early time, obtained from the first-order quantity $\Phi_{a}^{(1)}$ (see Eq. (2.7 for definition). The subsequent terms $P_{a b}^{1-\text { loop }}$ and $P_{a b}^{2-\text { loop }}$ represent the corrections to the linear-order perturbation, arising from the higher-order quantities, $\Phi_{a}^{(2)}, \Phi_{a}^{(3)}, \ldots$. In terms of the basic pieces of the diagrams shown in Fig. 1, the corrections $P_{a b}^{1-l o o p}(k)$ and $P_{a b}^{2 \text {-loop }}(k)$ can be diagrammatically written as the one-loop and twoloop diagrams, i.e., connected diagrams including one and two closed loops (e.g., see Fig.5 in Ref. 32]), and they are roughly proportional to $P_{0} \Delta_{0}^{2}$ and $P_{0} \Delta_{0}^{4}$, where $\Delta_{0}^{2}=k^{3} P_{0}(k) /\left(2 \pi^{2}\right)$. The explicit expressions for the power spectra together with the solutions of higher-order perturbation are summarized in Appendix $\mathrm{A}$.

It should be noted that in the standard PT expansion, the positivity of the perturbative corrections is not guaranteed. As we show later, the one- and two-loop contributions change the sign depending on the scale, and the absolute values of their amplitudes become comparable at lower redshift. In this respect, the standard PT has a poor convergence property, and the improvement of PT predictions may not be always guaranteed even including the higher-order corrections.

By contrast, renormalized $\mathrm{PT}^{2}$ re-organizes the naive expansions of the standard $\mathrm{PT}$ by introducing the nonperturbative statistical quantities [32]. In terms of these quantities, partial resummation of the naive expansion series is made, and the resultant convergence of the expansions is dramatically improved. In the renormalized PT, the power spectrum $P_{a b}(k ; \eta)$ is expressed in the form as

$$
P_{a b}(k ; \eta)=G_{a c}\left(k \mid \eta, \eta_{0}\right) G_{b d}\left(k \mid \eta, \eta_{0}\right) P_{c d}\left(k ; \eta_{0}\right)+P_{a b}^{(\mathrm{MC})}\left(k ; \eta, \eta_{0}\right)
$$

with $\eta_{0}$ being the time at which initial condition is imposed. Here, $P_{c d}\left(k ; \eta_{0}\right)$ is the power spectrum given at an early time $\eta_{0}$. The quantity $G_{a b}$ is one of the non-perturbative statistical quantities called non-linear propagator, together with the non-linear power spectrum. It is defined by

$$
\left\langle\frac{\delta \Phi_{a}(\boldsymbol{k} ; \eta)}{\delta \Phi_{b}\left(\boldsymbol{k}^{\prime} ; \eta^{\prime}\right)}\right\rangle=\delta_{\mathrm{D}}\left(\boldsymbol{k}-\boldsymbol{k}^{\prime}\right) G_{a b}\left(|\boldsymbol{k}| \mid \eta, \eta^{\prime}\right) ; \quad \eta \geq \eta^{\prime},
$$

where $\delta$ stands for a functional derivative. The propagator $G_{a b}$ describes the influence of an infinitesimal disturbance for $\Phi_{a}\left(\boldsymbol{k}^{\prime} ; \eta^{\prime}\right)$ on $\Phi_{a}(\boldsymbol{k} ; \eta)$, and it coincides with the linear propagator $g_{a b}$ in the limit $k \rightarrow 0$. Note that there is another non-perturbative statistical quantity called full vertex, which is the non-linear counterpart of the vertex function $\gamma_{a b c}$ [32].

\footnotetext{
${ }^{1}$ Strictly speaking, this statement is valid only when the universe at an early time is approximately described by the Einstein-de Sitter universe

2 In this paper, we intend to make a clear distinction between the terms 'renormalized PT' and 'RPT'. While the renormalized PT indicates the general non-perturbative formalism developed by Ref. [32], the RPT is meant to imply the practical approximation method for computing the power spectrum based on the renormalized PT, which has been developed by Ref. [34] (see Appendix B]).
} 
In the expression (3.4), the term $P_{a b}^{(\mathrm{MC})}$ represents the corrections coming from the loop diagrams. In contrast to the standard PT, the loop diagrams in $P_{a b}^{(\mathrm{MC})}$ are whole irreducible, as the result of renormalization or re-organization. Further, each of the irreducible diagrams consists of the non-perturbative quantities of non-linear power spectrum, non-linear propagator and full vertex. In this respect, renormalized PT is a fully non-perturbative formulation, and even the expansions truncated at some levels still contain the higher-order effects of non-linear gravitational evolution. This is the basic reason why the convergence properties in the renormalized PT are expected to be improved. As a trade-off, however, a straightforward application of renormalized PT seems difficult because of its non-perturbative formulation. While the term $P_{a b}^{\mathrm{MC}}$ collects only the irreducible diagrams, it is expressed as an infinite sum of the loop diagrams, each of which involve the non-linear power spectrum itself. In practice, the approximation or simplification is needed to evaluate the expressions (3.4), which we will discuss in next subsection.

\section{B. Closure approximation}

In this subsection, taking a great advantage of the formulation of renormalized PT, we discuss how to approximately treat Eq. (3.4) without losing its non-perturbative aspect as much as possible.

In the framework of renormalized PT, the non-perturbative effects on the power spectrum are largely attributed to the non-linear propagator. Thus, it seems essential to give a framework to treat both the non-linear propagator and power spectrum on an equal footing. As it has been pointed out by Ref. [32], a similar kind of the renormalized expansion to the power spectrum (3.4) can be made for the non-linear propagator:

$$
G_{a b}\left(k \mid \eta, \eta^{\prime}\right)=g_{a b}\left(\eta, \eta^{\prime}\right)+G_{a b}^{(\mathrm{MC})}\left(k ; \eta, \eta^{\prime}\right),
$$

where the term $G_{a b}^{(\mathrm{MC})}$ represents the mode-coupling correction, which is also made of the infinite sum of irreducible loop diagrams.

In order to give a self-consistent treatment for both Eqs. (3.4) and (3.6), a simple but transparent approach is to first (i) adopt the tree-level approximation of the full vertex function, and to (ii) apply the truncation procedure to the mode-coupling terms. This treatment has been frequently used in the statistical theory of turbulence in order to deal with the Navier-stokes equation, and is called closure approximation [43]. In the first approximation (i), the full vertex function is simply replaced with the linear-order one, i.e., $\gamma_{a b c}$ defined in Eq. (2.4). As for the truncation (ii), the simplest choice is to keep the one-loop renormalized diagram only, and to discard all other contributions.

With this approximation, the mode-coupling terms in $P_{a b}$ and $G_{a b}$ are simply described by $P_{a b}^{(\mathrm{MC})} \simeq P_{a b}^{(\mathrm{MC}, 1 \text {-loop })}$ and $G_{a b}^{(\mathrm{MC})} \simeq G_{a b}^{(\mathrm{MC}, 1-\mathrm{loop})}$. The analytical expressions for the one-loop contributions becomes [43]

$$
\begin{aligned}
P_{a b}^{(\mathrm{MC}, 1-\text { loop })}\left(k ; \eta, \eta^{\prime}\right)= & \int_{\eta_{0}}^{\eta} d \eta_{1} \int_{\eta_{0}}^{\eta^{\prime}} d \eta_{2} G_{a c}\left(k \mid \eta, \eta_{1}\right) G_{b d}\left(k \mid \eta^{\prime}, \eta_{2}\right) \Phi_{c d}\left(k ; \eta_{2}, \eta_{1}\right), \\
G_{a b}^{(\mathrm{MC}, 1-\text { loop })}\left(k ; \eta, \eta^{\prime}\right)= & \int_{\eta_{1}}^{\eta} d \eta_{1} \int_{\eta^{\prime}}^{\eta_{1}} d \eta_{2} g_{a c}\left(\eta, \eta_{1}\right) G_{s b}\left(k \mid \eta_{2}, \eta^{\prime}\right) \\
& \times 4 \int \frac{d^{3} \boldsymbol{q}}{(2 \pi)^{3}} \gamma_{c p q}(\boldsymbol{q}, \boldsymbol{k}-\boldsymbol{q}) P_{p r}\left(q ; \eta_{1}, \eta_{2}\right) G_{q l}\left(\mid \boldsymbol{k}-\boldsymbol{q} \| \eta_{1}, \eta_{2}\right) \gamma_{l r s}(-\boldsymbol{q}, \boldsymbol{k}) .
\end{aligned}
$$

The integrand in $P_{a b}^{(\mathrm{MC}, 1-\mathrm{loop})}$ contain the function $\Phi\left(k ; \eta_{1}, \eta_{2}\right)$, which represents the non-linear mode-coupling between different Fourier modes, given by

$$
\begin{aligned}
& \Phi_{a b}\left(k ; \eta_{1}, \eta_{2}\right)=2 \int \frac{d^{3} \boldsymbol{q}}{(2 \pi)^{3}} \gamma_{a r s}(\boldsymbol{q}, \boldsymbol{k}-\boldsymbol{q}) \gamma_{b p q}(\boldsymbol{q}, \boldsymbol{k}-\boldsymbol{q}) \\
& \quad \times\left\{P_{p r}\left(q ; \eta_{1}, \eta_{2}\right) P_{q s}\left(|\boldsymbol{k}-\boldsymbol{q}| ; \eta_{1}, \eta_{2}\right) \Theta\left(\eta_{1}-\eta_{2}\right)+P_{r p}\left(q ; \eta_{2}, \eta_{1}\right) P_{s q}\left(|\boldsymbol{k}-\boldsymbol{q}| ; \eta_{2}, \eta_{1}\right) \Theta\left(\eta_{2}-\eta_{1}\right)\right\} .
\end{aligned}
$$

Note that the mode-coupling function $\Phi$ possesses the following symmetry: $\Phi_{a b}\left(k ; \eta_{1}, \eta_{2}\right)=\Phi_{b a}\left(k ; \eta_{2}, \eta_{1}\right)$. The corresponding diagrams to the integral expressions for power spectrum and non-linear propagator, i.e., Eqs. (3.4) and (3.6) with mode-coupling terms (3.7) and (3.8), are shown in Fig. 2.

It is worth mentioning that the integral equations (3.4) and (3.6) with truncated mode-coupling terms (3.7) and (3.8) can be recast in the form of the integro-differential equations, and both the power spectrum and non-linear propagator can be computed by solving the evolution equations. This forward treatment seems especially suited for the full non-linear treatment of closure approximation and would be faster than directly treating the integral 


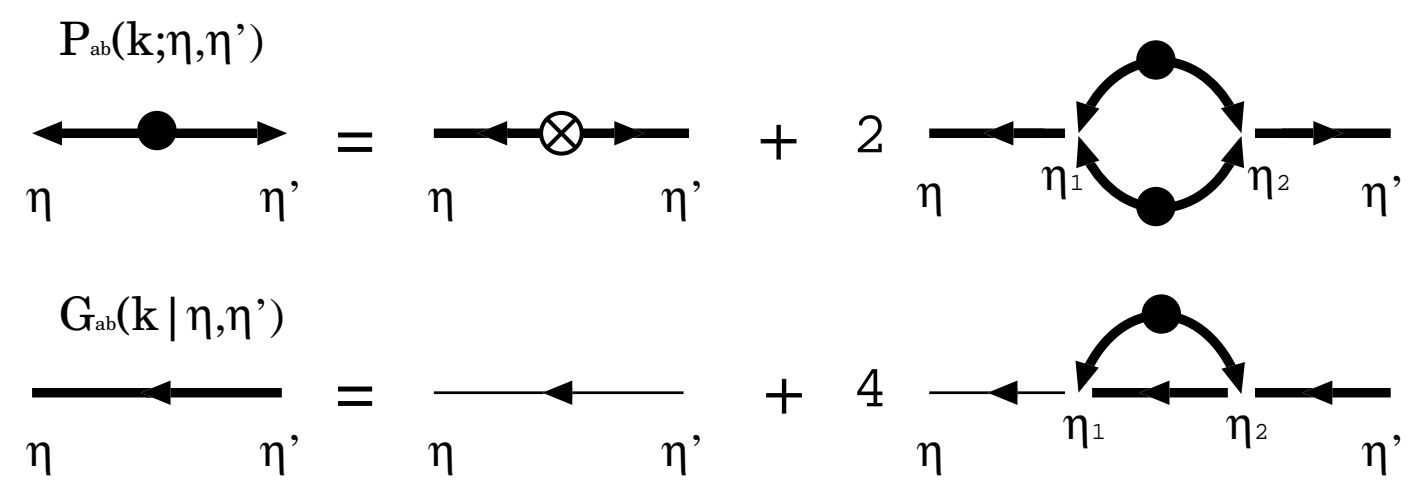

FIG. 2: Diagrammatic representation of the power spectrum and non-linear propagator in closure approximation. The thick lines represent the full-order quantities, while the thin line indicates the linear-order one. The second terms at right-hand side indicate the irreducible one-loop diagrams of the mode-coupling terms, $P_{a b}^{(\mathrm{MC}, 1-\mathrm{loop})}$ and $G_{a b}^{(\mathrm{MC}, 1-\mathrm{loop})}$. In the renormalized PT, the mode-coupling term is expressed as an infinite sum of the irreducible loop corrections. Truncating the infinite sum at one-loop order and adopting the tree-level approximation of the full vertex function, we obtain the closed system of power spectrum and propagator, as shown in the figure.

equations. Numerical algorithm to solve evolution equations, together with preliminary results, is presented in details in Ref. [48] (see also [49]).

In the present paper, we are especially concerned with the evolution of BAOs around $k \lesssim 0.4 h \mathrm{Mpc}^{-1}$, where the nonlinearity of gravitational clustering is rather mild, and the analytical treatment even involving some approximations is still useful. Here, employing the Born approximation, we analytically evaluate the integral equations (3.4) and (3.7) [43]. A fully numerical study on BAOs without Born approximation will be discussed in a separate paper.

The Born approximation is the iterative approximation scheme in which the leading-order solutions are first obtained by replacing the quantities in the non-linear integral terms with linear-order ones. The solutions can be improved by repeating the iterative substitution of the leading-order solutions into the non-linear integral terms. Consider the time evolution of the power spectrum started from the time $\eta_{0}$. For a sufficiently small value of $\eta_{0}$, the early-time evolution of power spectrum is well-approximated by the linear theory. Assuming the growing-mode initial condition, we have

$$
P_{a b}\left(k ; \eta_{0}\right)=e^{2 \eta_{0}} u_{a} u_{b} P_{0}(k)
$$

with $u_{a}=(1,1)$. Then, substituting Eq. (3.10) into (3.4), the iterative evaluation of the the integral equations (3.4) with (3.7) by the Born approximation leads to [43]

$$
P_{a b}(k ; \eta)=\widetilde{G}_{a}\left(k \mid \eta, \eta_{0}\right) \widetilde{G}_{b}\left(k \mid \eta, \eta_{0}\right) e^{2 \eta_{0}} P_{0}(k)+P_{a b}^{(\mathrm{MC} 1)}(k ; \eta)+P_{a b}^{(\mathrm{MC} 2)}(k ; \eta)+\cdots,
$$

where we define $\widetilde{G}_{a} \equiv G_{a 1}+G_{a 2}$. The terms $P_{a b}^{(\mathrm{MC} 1)}$ and $P_{a b}^{(\mathrm{MC} 2)}$ respectively represent the leading- and next-to-leading order results of the Born approximation to the mode-coupling term (3.7). The explicit expressions become

$$
\begin{gathered}
P_{a b}^{(\mathrm{MC} 1)}(k ; \eta)=2 \int \frac{d^{3} \boldsymbol{q}}{(2 \pi)^{3}} I_{a}\left(\boldsymbol{k}, \boldsymbol{q} ; \eta, \eta_{0}\right) I_{b}\left(\boldsymbol{k}, \boldsymbol{q} ; \eta, \eta_{0}\right) e^{4 \eta_{0}} P_{0}(q) P_{0}(|\boldsymbol{k}-\boldsymbol{q}|), \\
P_{a b}^{(\mathrm{MC} 2)}(k ; \eta)=8 \int \frac{d^{3} \boldsymbol{p}}{(2 \pi)^{3}} \int \frac{d^{3} \boldsymbol{q}}{(2 \pi)^{3}} J_{a}\left(\boldsymbol{k}, \boldsymbol{p}, \boldsymbol{q} ; \eta, \eta_{0}\right) J_{b}\left(\boldsymbol{k}, \boldsymbol{p}, \boldsymbol{q} ; \eta, \eta_{0}\right) \\
\times e^{6 \eta_{0}} P_{0}(|\boldsymbol{k}-\boldsymbol{p}|) P_{0}(q) P_{0}(|\boldsymbol{p}-\boldsymbol{q}|) .
\end{gathered}
$$

The kernels $I_{a}$ and $J_{a}$ are respectively given by

$$
\begin{aligned}
I_{a}\left(\boldsymbol{k}, \boldsymbol{q} ; \eta, \eta_{0}\right)=\int_{\eta_{0}}^{\eta} d \eta^{\prime} G_{a l}\left(k \mid \eta, \eta^{\prime}\right) \gamma_{l r s}(\boldsymbol{q}, \boldsymbol{k}-\boldsymbol{q}) \widetilde{G}_{r}\left(q \mid \eta^{\prime}, \eta_{0}\right) \widetilde{G}_{s}\left(\mid \boldsymbol{k}-\boldsymbol{q} \| \eta^{\prime}, \eta_{0}\right), \\
\begin{aligned}
J_{a}\left(\boldsymbol{k}, \boldsymbol{p}, \boldsymbol{q} ; \eta, \eta_{0}\right)= & \int_{\eta_{0}}^{\eta} d \eta_{1} \int_{\eta_{0}}^{\eta} d \eta_{2} G_{a l}\left(k \mid \eta, \eta_{1}\right) \gamma_{l r s}(\boldsymbol{p}, \boldsymbol{k}-\boldsymbol{p}) G_{r c}\left(p \mid \eta_{1}, \eta_{2}\right) \\
& \times \gamma_{c p q}(\boldsymbol{q}, \boldsymbol{p}-\boldsymbol{q}) \widetilde{G}_{p}\left(q \mid \eta_{2}, \eta_{0}\right) \widetilde{G}_{q}\left(\mid \boldsymbol{p}-\boldsymbol{q} \| \eta_{2}, \eta_{0}\right) \widetilde{G}_{s}\left(\mid \boldsymbol{k}-\boldsymbol{p} \| \eta_{1}, \eta_{0}\right) .
\end{aligned}
\end{aligned}
$$




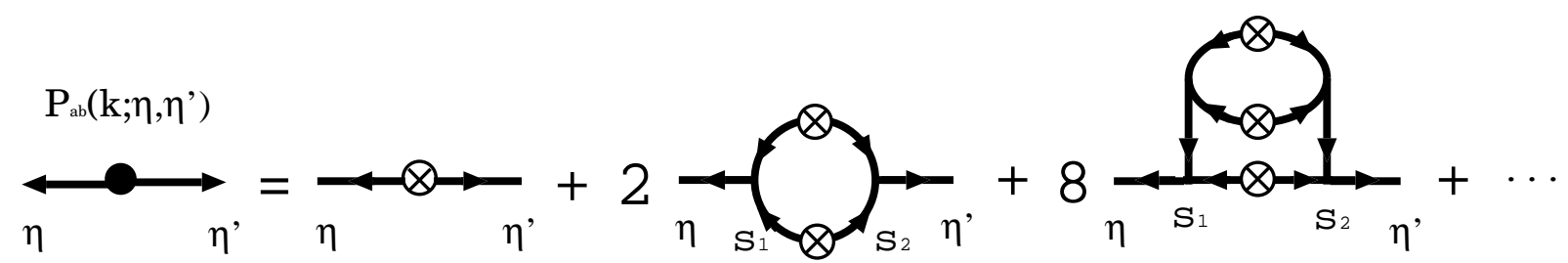

FIG. 3: Diagrammatic representation for the perturbative treatment of the power spectrum with the Born approximation, i.e., Eq. (3.11).

The diagram corresponding to the above expressions is shown in Fig. 3. Note that in deriving the expression (3.11), we do not expand the propagators $G_{a b}$ and their non-perturbative properties still hold. In order to evaluate Eq. (3.11), we use the analytic solution of $G_{a b}$ derived in Ref. [43], where the non-linear propagator was constructed approximately by matching the asymptotic behaviors at low- and high-k modes, based on Eqs. (3.6) with (3.8). The resultant analytic solution behaves like $G_{a b} \rightarrow g_{a b} J_{1}(2 x) / x$ at $k \rightarrow \infty$, where the quantity $J_{1}$ is the Bessel function with its argument $x=k \sigma_{\mathrm{v}}\left(e^{\eta}-e^{\eta^{\prime}}\right)$, and the velocity dispersion $\sigma_{\mathrm{v}}$ is approximately described by the linear theory, i.e., $\sigma_{\mathrm{v}}^{2} \simeq \sigma_{\mathrm{v}, \text { lin }}^{2}=\int d q P_{\operatorname{lin}}(q ; z) /\left(6 \pi^{2}\right)$. Note that the final results of the power spectrum are a little bit sensitive to the high- $k$ behavior of the propagator, and a naive application of the approximate solution leads to a slight shift in the amplitude of power spectrum. While this is not serious at all for the leading-order calculation, it amounts a percent-level shift when we consider the higher-order correction, $P_{a b}^{(\mathrm{MC} 2)}$. As discussed by Crocce \& Scoccimarro (2008), one possible reason for this may be a small contribution from the sub-leading corrections in the propagator. In order to remedy the effect of small corrections, we follow the method proposed by Ref. [34]. We define

$$
\alpha(z) \equiv\left[\frac{\int_{0}^{k_{\max }} d q P_{\mathrm{nl}}(q ; z)}{\int_{0}^{k_{\max }} d k P_{\operatorname{lin}}(k ; z)}\right]^{1 / 2},
$$

where $P_{\mathrm{nl}}$ means the non-linear matter power spectrum. Then, the sub-leading correction can be corrected by simply multiplying the factor $\alpha$ by $\sigma_{\mathrm{v}}$, i.e., $\sigma_{\mathrm{v}} \rightarrow \alpha(z) \sigma_{\mathrm{v}}$. Note that this treatment is only applied to the propagator in the lowest-order term in Eq. (3.11), which most sensitively affects the power spectrum amplitude on small scales. For simplicity, we use halofit [50] to compute $P_{\mathrm{nl}}$ and adopt the cutoff wavenumber, $k_{\max }=k_{\sigma}$, where $k_{\sigma}$ is the non-linear scale defined by Ref. [50].

In the rest of this paper, we present the results for the analytic treatment based on the expression (3.11). In computing the mode-coupling terms $P_{a b}^{(\mathrm{MC} 1)}$ and $P_{a b}^{(\mathrm{MC} 2)}$, we must first evaluate the functions $I_{a}$ and $J_{a}$ for a given set of arguments, which involve the one- and two-dimensional integrals over time $\eta$. We use the Gaussian quadrature for these time integrations. As for the momentum integrals in the mode-coupling terms, thanks to the symmetry of the functions $I_{a}$ and $J_{a}$, the multi-dimensional integrals in $P_{a b}^{(\mathrm{MC} 1)}$ and $P_{a b}^{(\mathrm{MC} 2)}$ can be reduced to the two- and four-dimensional integrals, respectively. We use the Gaussian quadratures for the momentum integral in $P_{a b}^{(\mathrm{MC} 1)}$. The four-dimensional momentum integration in the mode-coupling term $P_{a b}^{(\mathrm{MC} 2)}$ is performed with Monte Carlo technique of quasi-random sampling using the library, Cuba $[51]^{3}$.

Finally, we note that the formulation and analytic treatment presented here have several distinctions and similarities to the other non-perturbative calculations proposed recently. In Appendix B, we compare the present work with a subset of these treatments, and discuss how the approach developed here is complementary to or expands on these studies.

\section{IMPROVED PT VS. NUMERICAL SIMULATIONS}

In this section, particularly focusing on the BAOs, we compare the improved PT predictions from the analytic treatment of closure approximation with results of N-body simulations.

\footnotetext{
3 http://www.feynarts.de/cuba/
} 


\section{A. N-body simulations}

We use a publicly available cosmological N-body code, Gagdet2 [52]. We ran two sets of simulations, wmap3 and wmap5, in which we adopt the standard Lambda CDM model with cosmological parameters determined from the WMAP3 and WMAP5, respectively [2, 3]. The wmap3 run is basically the same N-body run as described in Ref. [45], and a quantitative comparison between the leading-order results of improved PT and simulations has been previously made. We basically use the results of wmap3 run to check the consistency of the present calculations with the previous work. The wmap3 run is also helpful to cross-check the convergence properties in the new simulation, wmap5, which increase the number of realizations to 30 . Table [ summarizes the parameters used in the simulations. The initial conditions were created with the 2LPT code [53] at initial redshift $z_{\text {ini }}=31$, based on the linear transfer function calculated from CAMB [54]. The number of meshes used in the particle-mesh computation is $1,024^{3}$. We adopt a softening length of $0.1 h^{-1} \mathrm{Mpc}$ for tree forces.

We store three output redshifts for wmap3 run, whereas we select four output redshifts for wmap5 run; $z=3,1$, and 0 (wmap3) : $z=3,2,1$, and 0.5 (wmap5). Using these outputs, we compute the power spectrum and two-point correlation function in both real and redshift spaces.

The calculation of the matter power spectrum adopted here is basically the same treatment as in Ref. [45]. The standard method to compute the power spectrum is to square the Fourier transform of the density field and to take an average over realizations and Fourier modes. This is given by

$$
\widehat{P}\left(k_{n}\right)=\frac{1}{N_{n}^{\mathrm{k}} N^{\text {run }}} \sum_{m=1}^{N^{\text {run }}} \sum_{k_{n}^{\min }<|\boldsymbol{k}|<k_{n}^{\max }}\left|\delta^{m-\mathrm{th}}(\boldsymbol{k})\right|^{2} ; \quad k_{n} \equiv \frac{1}{N_{n}^{\mathrm{k}}} \sum_{k_{n}^{\min }<|\boldsymbol{k}|<r_{k}^{\max }}|\boldsymbol{k}|,
$$

where $N_{n}^{\mathrm{k}}$ and $N^{\text {run }}$ are the number of Fourier modes in the $n$-th wavenumber bin and the number of realizations, and $k_{n}^{\min }$ and $k_{n}^{\max }$ are the minimum and the maximum wavenumber of the $n$-th bin, respectively. The quantity $\delta^{m \text {-th }}(\boldsymbol{k})$ means the density field in Fourier space obtained from the $m$-th realization data. We use the Cloud-in-Cells interpolation for the density assignment of particles onto a $1,024^{3}$ mesh, and correct the window function. Note that the power spectra measured from the standard treatment above suffer from the effect of finite-mode sampling discussed by Ref. [55]. The resultant power spectrum deviates from the prediction for the ideal ensemble average, and exhibits the anomalous growth of power spectrum amplitude on large scales. In order to reduce the effect of finite mode sampling at $k \lesssim 0.1 \mathrm{~h} \mathrm{Mpc}^{-1}$, we multiply the measured power spectrum by the ratio, $\widehat{P}^{\mathrm{PT}}(k) / P_{\text {lin }}(k)$, where the quantity $\widehat{P}^{\mathrm{PT}}(k)$ is calculated from the perturbation theory up to the third-order in density field, and $P_{\text {lin }}(k)$ is the input linear power spectrum extrapolated to a given output redshift. Note that in computing $\widehat{P}^{\mathrm{PT}}(k)$, we use the Gaussian-sampled density field used to generate the initial condition of each N-body run. With this treatment, the individual random nature of each N-body run is weakened, and the errors associated with anomalous growth is reduced ${ }^{4}$.

For the estimation of two-point correlation function, we adopt the grid-based calculation using the Fast Fourier Transformation (FFT). In this treatment, similar to the power spectrum analysis, we first compute the square of the density field on each grid of Fourier space. Then, applying the inverse Fourier transformation, we take the average over realization and distance, and obtain the two-point correlation function. Schematically, this is expressed as

$$
\widehat{\xi}\left(r_{n}\right)=\frac{1}{N_{n}^{\mathrm{r}} N^{\text {run }}} \sum_{m=1}^{N^{\text {run }}} \sum_{r_{n}^{\min }<|\boldsymbol{r}|<r_{n}^{\max }} \widehat{\mathrm{FFT}}^{-1}\left[\left|\delta^{m-\text { th }}(\boldsymbol{k})\right|^{2} ; \boldsymbol{r}\right],
$$

where the operation $\widehat{\mathrm{FFT}}^{-1}$ stands for the inverse FFT of the squared density field on each grid. Note here that $r_{n}$ is simply chosen at the center of the $n$-th radial bin, i.e., $r_{n}=\left(r_{\min }+r_{\max }\right) / 2$.

Eq. (4.2) usually suffers from the ambiguity of the zero-point normalization in the amplitude of two-point correlation function, because of the lack of the low- $k$ powers due to the finite boxsize of the simulations. With the $1,024^{3}$ grids and the boxsize of $L_{\mathrm{box}}=1 h^{-1} \mathrm{Gpc}$, however, we can safely evaluate the two-point correlation function around the

\footnotetext{
${ }^{4}$ In Ref. [45], the correction to the effect of finite-mode sampling has been applied to the real-space power spectra. Here, we extend it to compute the redshift-space power spectrum by simply replacing the ratio $\widehat{P}^{\mathrm{PT}}(k) / P_{\text {lin }}(k)$ with that in redshift space. To be precise, we compute the multipole moments of the redshift-space power spectrum, and the ratio, $\widehat{P}_{\ell}^{(\mathrm{S}), \mathrm{PT}}(k) / P_{\ell, \text { lin }}^{(\mathrm{S})}(k)$, is multiplied for each multipole spectrum (see Sec. IV C 1).
} 
TABLE I: Parameters of N-body simulations

\begin{tabular}{ccccc|cccccc}
\hline \hline Name & $L_{\text {box }}$ & \# of particles & $z_{\text {ini }}$ & \# of runs & $\Omega_{\mathrm{m}}$ & $\Omega_{\Lambda}$ & $\Omega_{\mathrm{b}} / \Omega_{\mathrm{m}}$ & $h$ & $n_{s}$ & $\sigma_{8}$ \\
\hline wmap3 & $1000 h^{-1} \mathrm{Mpc}$ & $512^{3}$ & 31 & 4 & 0.234 & 0.766 & 0.175 & 0.734 & 0.961 & 0.76 \\
wmap5 & $1000 h^{-1} \mathrm{Mpc}$ & $512^{3}$ & 31 & 30 & 0.279 & 0.721 & 0.165 & 0.701 & 0.96 & 0.817 \\
\hline \hline
\end{tabular}
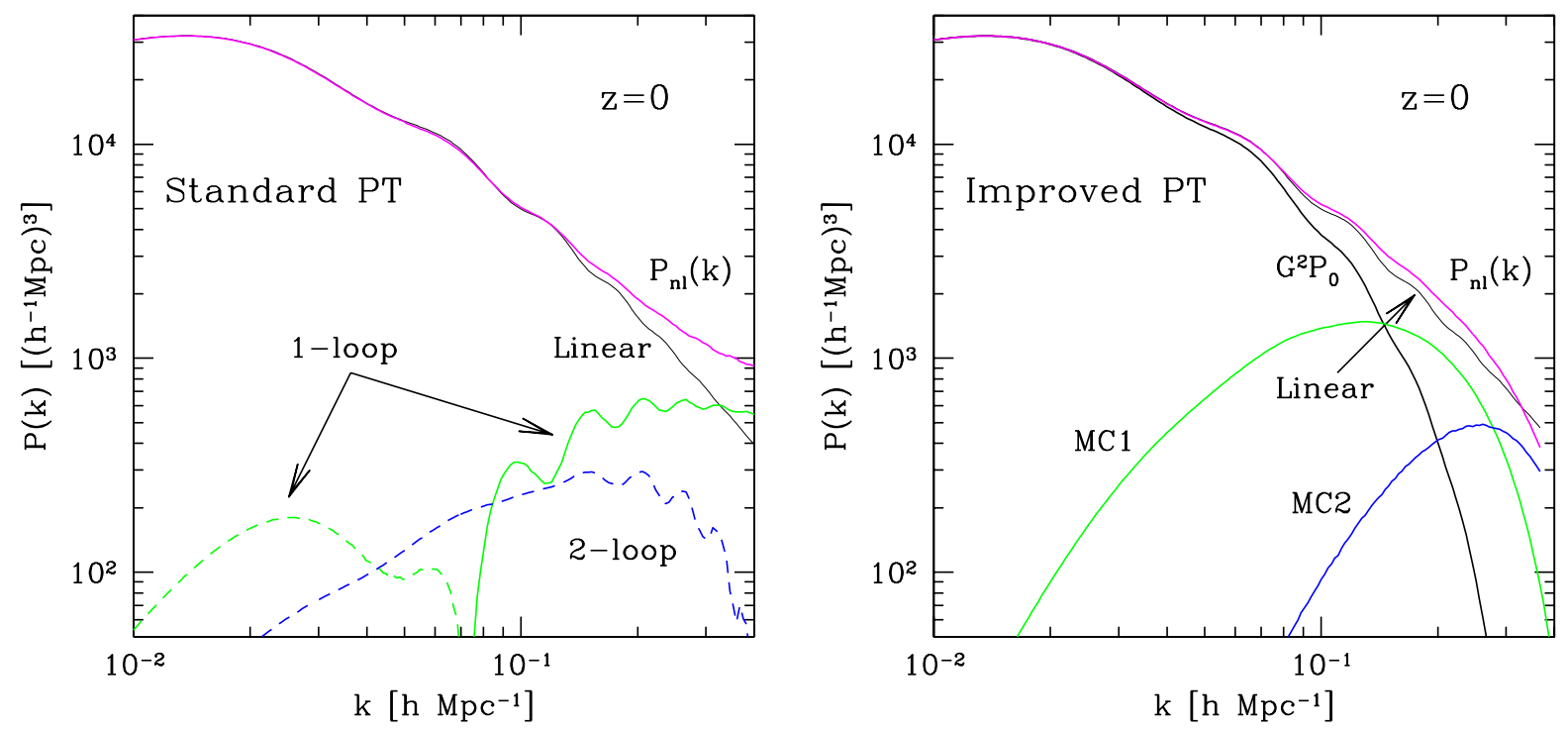

FIG. 4: Convergence properties of standard PT (left) and improved PT (right) expansions in the matter power spectrum. In each panel, the higher-order contributions to the total power spectrum labeled as $P_{\mathrm{nl}}$ is separately plotted. In left panel, one-loop and two-loop corrections in the standard PT, $P_{11}^{1-\text { loop }}$ and $P_{11}^{2-\text { loop }}$, are plotted, while in right panel, the mode-coupling corrections $P_{11}^{(\mathrm{MC} 1)}$ and $P_{11}^{(\mathrm{MC} 2)}$ in the improved PT given at Eqs. (3.12) and (3.13) respectively shown (labeled as MC1 and $\mathrm{MC2}$ ), together with the first term in Eq. (3.11) (labeled as $\mathrm{G}^{2} \mathrm{P}_{0}$ ). Note that dashed lines indicate the negative values.

baryon acoustic peak. Comparison between different computational methods, together with convergence check of this method, is presented in Appendix C

Finally, similar to the estimation of power spectrum, the finite-mode sampling also affects the calculation of the two-point correlation function. We thus correct it by subtracting and adding the extrapolated linear density field as, $\widehat{\xi}(r)-\widehat{\xi}_{\text {lin }}(r)+\xi_{\text {lin }}(r)$, where $\widehat{\xi}_{\text {lin }}$ is the correlation function estimated from the Gaussian density field, and $\xi_{\text {lin }}$ is the linear theory prediction of two-point correlation function.

\section{B. Results in real space}

\section{Power spectrum}

Before addressing a quantitative comparison between N-body simulation and improved PT, we first discuss the convergence properties of the improved PT, and consider how well the calculation based on the improved PT does improve the prediction compared to the standard PT.

Fig. 4 4 plots the overall behaviors of the non-linear power spectrum of density fluctuation, $P(k ; z) \equiv P_{11}(k ; z)$, given at $z=0$, adopting the wmap3 cosmological parameters. In left panel, the results of standard PT are shown, and the contributions to the total power spectrum up to the two-loop diagrams are separately plotted. On the other hand, right panel shows the results of improved PT. We plot the contributions up to the second-order Born approximation labeled as MC1 and MC2.

In Fig. 4 there are clear distinctions between standard and improved PTs. While the loop corrections in standard PT change their signs depending on the scales and exhibit an oscillatory feature, the corrections coming from the Born 

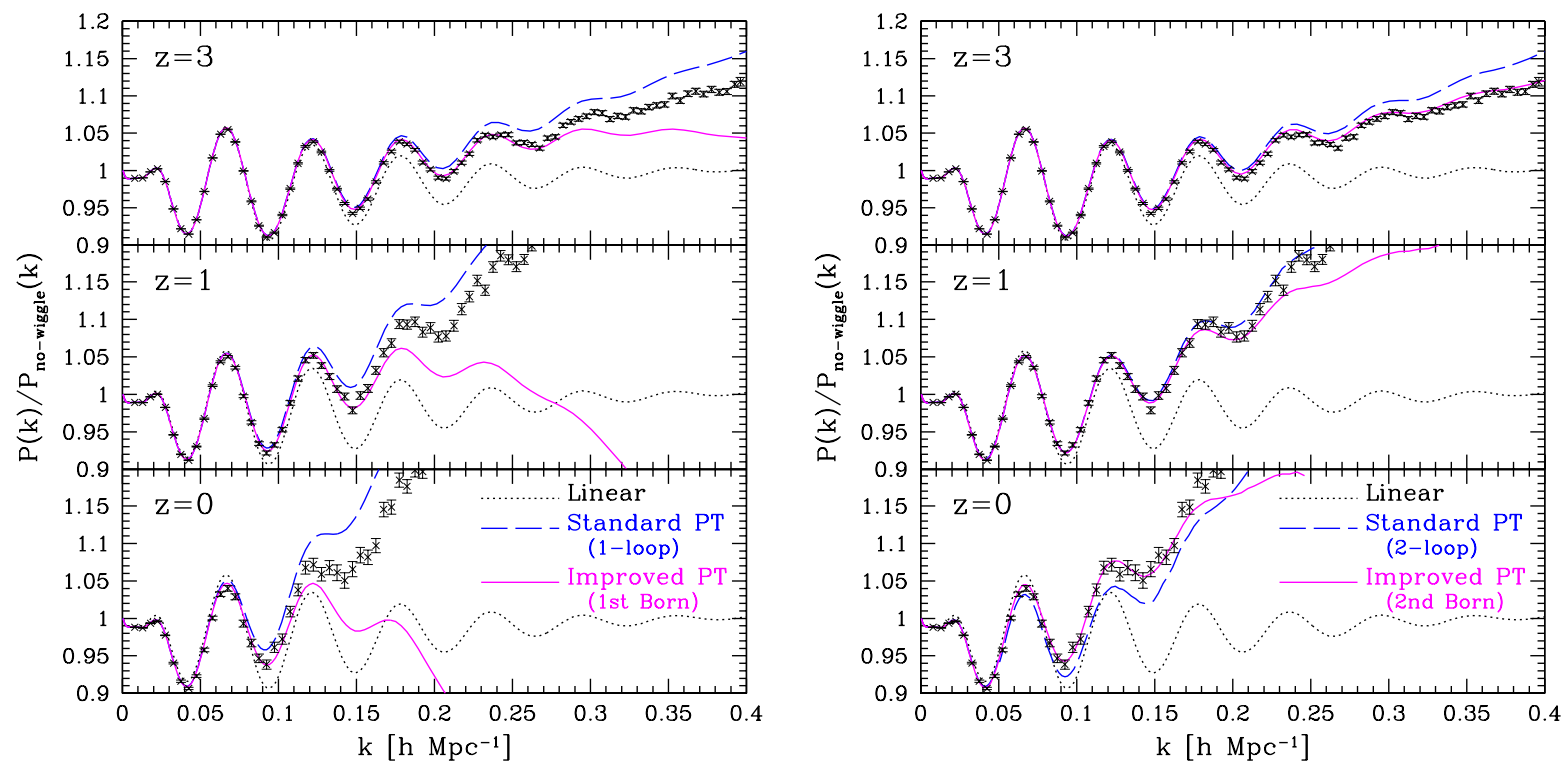

FIG. 5: Ratios of power spectrum to smoothed reference spectrum, $P(k) / P_{\text {no-wiggle }}(k)$, given at redshifts $z=3$ (top), 1 (middle) and 0(bottom). Cosmological parameters used in the wmap3 simulations are adopted to compute the power spectrum from standard PT and improved PT, and the results are compared with N-body simulations (symbols with error-bars). The reference spectrum $P_{\text {no-wiggle }}(k)$ is calculated from the no-wiggle formula of the linear transfer function in Ref. [23]. In each panel, dotted, dashed and solid lines represent the linear, standard PT and improved PT results, respectively. In left panel, leading-order results of standard PT and improved PT are shown, while in right panel, the results including the higher-order corrections are plotted.

approximation in the improved PT are all positive and mostly the smooth function of $k$. Further, the higher-order corrections in the improved PT have a remarkable scale-dependent property compared to those in the standard PT; their contributions are well-localized around some characteristic wavenumbers, and they are shifted to the higher $k$ modes as increasing the order of PT. These trends clearly indicate that the improved PT with closure approximation has a better convergence property. Qualitative behaviors of the higher-order corrections quite resemble the predictions of RPT by Crocce \& Scoccimarro (2008) [34].

Now, let us focus on the behavior of BAOs, and discuss how the convergence properties seen in Fig. 4)affect the predictions of BAO features. In Fig. 5, adopting the wmap3 cosmological parameters, we plot the ratio, $P(k) / P_{\text {no-wiggle }}(k)$, where the function $P_{\text {no-wiggle }}(k)$ is the linear power spectrum from the smooth transfer function neglecting the BAO feature in Ref. [23]. In left panel, N-body simulations are compared with the leading-order results of PT predictions, i.e., standard PT including the one-loop correction (dashed), and improved PT with first-order Born correction (solid). Apart from the wiggle structure, the amplitude of standard PT prediction monotonically increases with wavenumber $k$, and tends to overestimate the results of N-body simulations. On the other hand, the amplitude of improved PT prediction rapidly falls off at a certain wavenumber, and the deviation from N-body results becomes significant. However, a closer look at the behavior on large scales reveals that improved PT prediction gives a better agreement with simulation. The results are indeed consistent with the previous findings in Ref. [45]. The situation becomes more impressive when we add the next-to-leading order corrections. As shown in right panel, the improved PT gets the power on smaller scales, and reproduces the N-body results in a wider range of wavenumber. By contrast, the prediction of standard PT depicted as dashed lines seems a little bit subtle. Compared to the one-loop results, the amplitudes of the standard PT prediction including the two-loop correction are slightly reduced, and the agreement with N-body simulation seems apparently improved a bit at higher redshift. At lower redshift $z=0$, however, the correction coming from the two-loop order becomes significant, and the prediction eventually underestimates the simulation. The reason for these behaviors basically comes from the competition between positive and negative contributions of the one-loop and two-loop corrections, respectively (see left panel of Fig . 4). These are consistent with those findings in Ref. [46] (see Fig. 1 of their paper).

In Fig. 66, to clarify the range of agreement in more quantitative ways, we plot the fractional difference divided by the smoothed reference spectra, $\left[P_{\mathrm{N}-\text { body }}(k)-P_{\mathrm{PT}}(k)\right] / P_{\text {no-wiggle }}$, where the quantity $P_{\mathrm{PT}}(k)$ implies the standard and improved PT predictions in left and right panels, respectively. Here, the vertical arrows represent the maximum wavenumber $k_{1 \%}$, below which the leading-order predictions of standard or improved PT reproduce the N-body results 

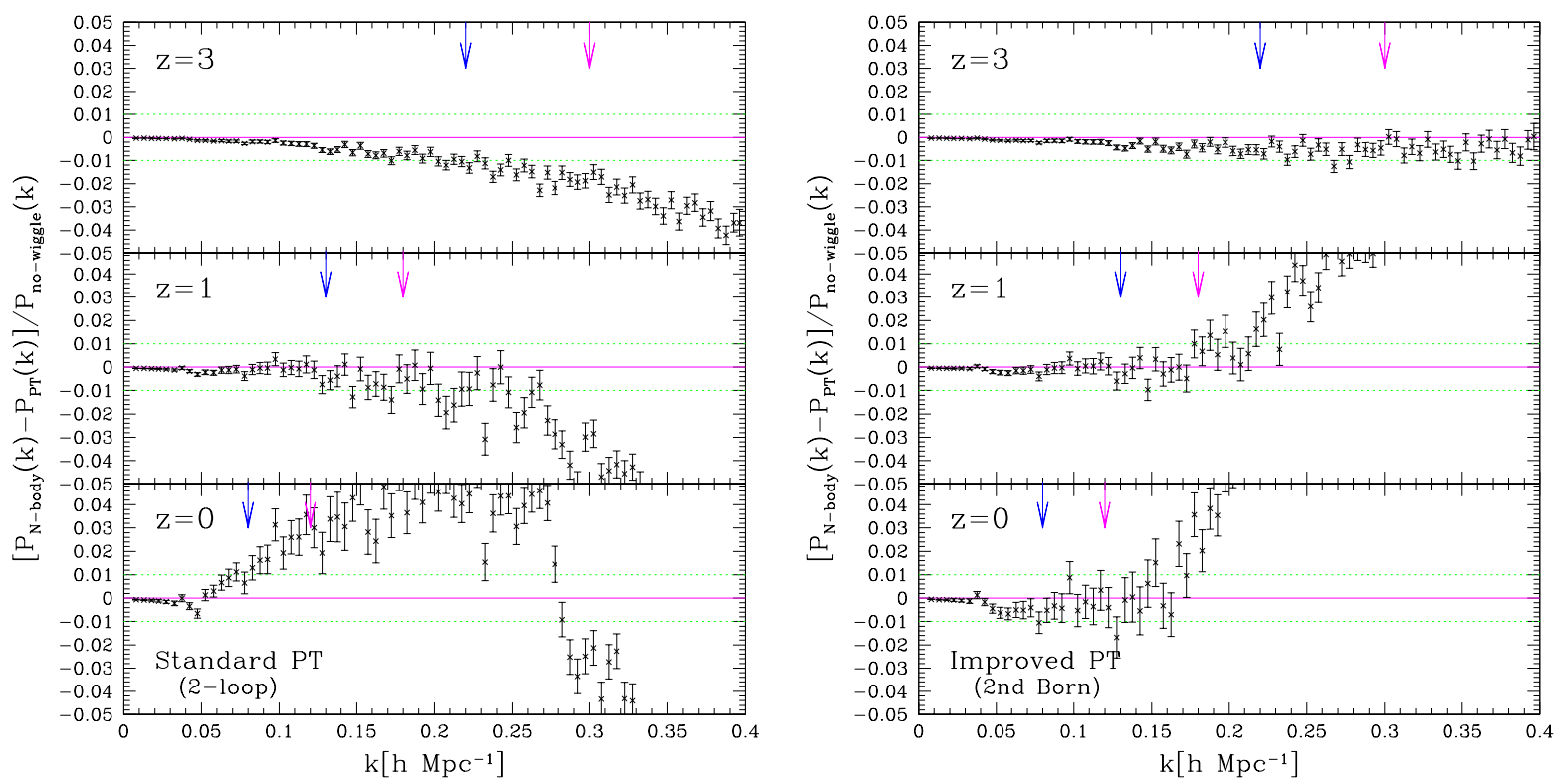

FIG. 6: Difference between N-body and PT results divided by the reference spectrum, $\left[P_{\mathrm{N}-\text { body }}(k)-P_{\mathrm{PT}}(k)\right] / P_{\text {no-wiggle }}(k)$. Left panel shows the results for standard PT up to the two-loop order. Right panel presents the case of improved PT including the corrections up to the second-order Born approximation of the mode-coupling term. In both panels, vertical arrows represent the wavenumbers $k_{1 \%}$ of standard and improved PT (from left to right), below which the leading-order PT predictions reproduce the N-body simulations well within $1 \%$ accuracy (see text in details).

quite well within the $1 \%$ accuracy. According to Nishimichi et al. [45], this has been determined by the detailed comparison between models and simulations, and is empirically characterized by solving the following equation:

$$
\frac{k_{1 \%}^{2}}{6 \pi^{2}} \int_{0}^{k_{1} \%} d q P_{\operatorname{lin}}(q ; z)=C
$$

with $C=0.18$ for the one-loop standard PT, and $C=0.35$ for the improved PT up to the first-order Born correction.

Comparing these convergence regimes of the leading-order calculation with results of fractional differences, Fig. 6 shows that the inclusion of higher-order terms does not always improve the prediction in the standard PT treatment. By contrast, the improved PT calculation does improve the predictions, and the range of agreement between N-body simulations and the predictions becomes wider.

In Fig. 7, we plot the results for the wmap5 simulations, which have relatively large value of $\sigma_{8}$ compared to the wmap3 run (see Table I). Left and right panels respectively plot the ratio of power spectrum amplitude and the fractional difference between N-body results and improved PT predictions. With the 30 runs of N-body simulations, the errors in the power spectrum amplitude are greatly reduced, and it is clearly shown that the predictions of improved PT including the higher-order corrections almost coincide with the N-body results beyond the convergence regime of the leading-order calculations (indicated by vertical arrows), and achieve a sub-percent accuracy. From this plot, the maximum wavenumber $k_{1 \%}$ at each redshift can be estimated by comparing the predictions with N-body results as $k_{1 \%}=0.20 \mathrm{~h} \mathrm{Mpc}^{-1}(\mathrm{z}=0.5), 0.23 h \mathrm{Mpc}^{-1}(\mathrm{z}=1), 0.33 \mathrm{hMpc}^{-1}(\mathrm{z}=2)$ and $0.47 \mathrm{hMpc}^{-1}(\mathrm{z}=3)$. These values roughly match those determined from the criterion (4.3) with the constant $C=0.70$.

Although we did not store the $z=0$ data of wmap5 run to compare with analytic prediction, Eq. (4.3) using this constant value implies that the maximum wavenumber for improved PT becomes $k_{1 \%}=0.15 h \mathrm{Mpc}^{-1}$, which contrasts with the one for the one-loop prediction of standard PT, $k_{1 \%}=0.09 \mathrm{hppc}^{-1}$. Thus, the improved PT including up to the second-order Born approximation is expected to be still accurate at $z=0$, and it can cover the major part of the BAOs. A more detailed comparison at low redshift including other analytic prescriptions can be found in Ref. [46]. 

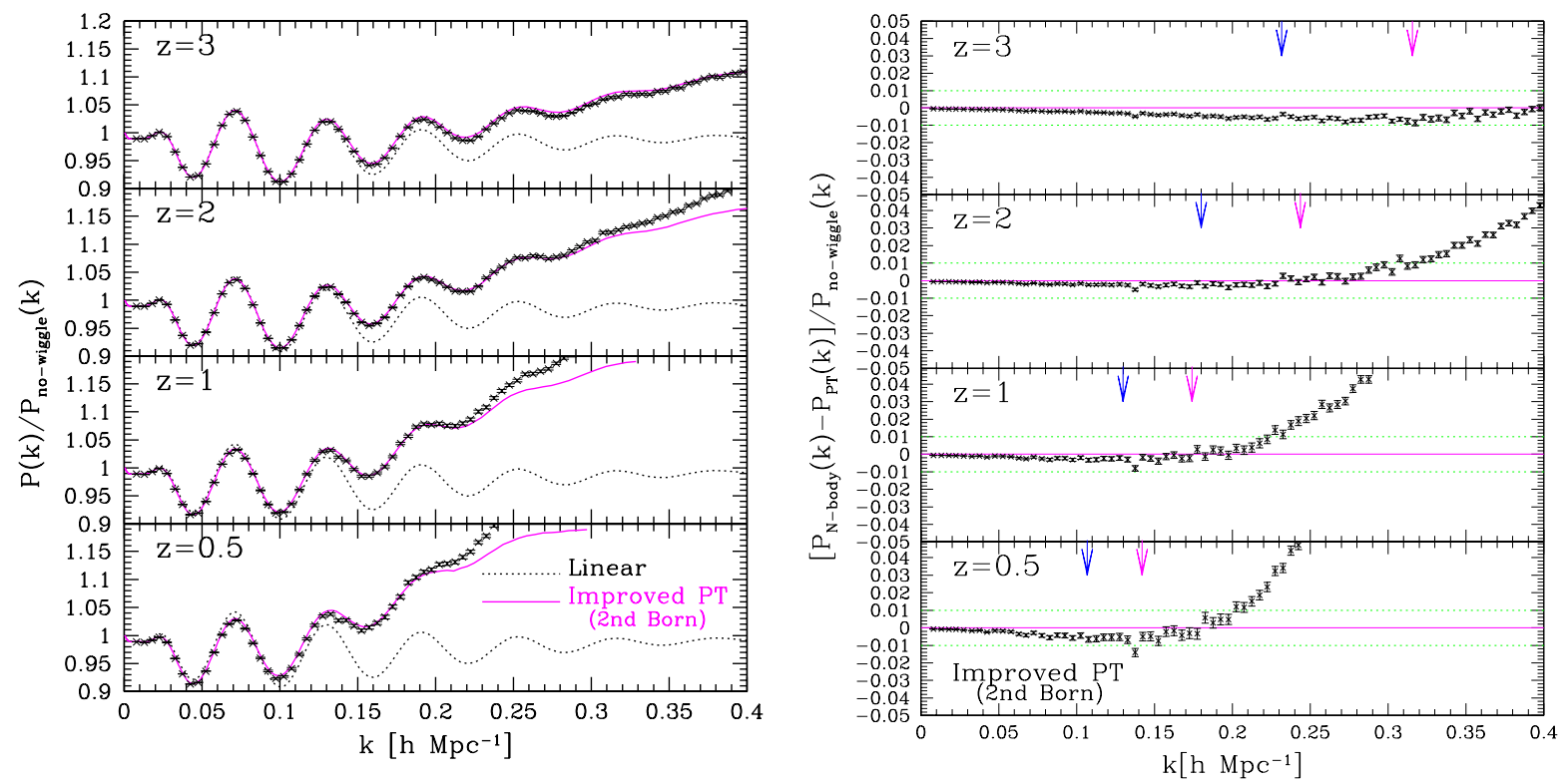

FIG. 7: Comparison between N-body results and improved PT predictions in the case adopting wmap5 cosmological parameters. From top to bottom, the results at $z=3,2,1$ and 0.5 are shown. The improved PT predictions plotted here include the corrections up to the second-order Born approximation of the mode-coupling term, $P^{\mathrm{MC} 2}$. Left: ratio of power spectrum to the smoothed reference spectra, $P(k) / P_{\text {no-wiggle }}(k)$. Solid and dotted lines are improved PT and linear theory predictions, respectively. Right: difference between N-body and improved PT results normalized by the no-wiggle formula, $\left[P_{\mathrm{N}-\text { body }}(k)-\right.$ $\left.P_{\mathrm{PT}}(k)\right] / P_{\text {no-wiggle }}(k)$. In each panel, vertical arrows represent the wavenumber $k_{1 \%}$ for the leading-order predictions of standard and improved PT (from left to right).

\section{Correlation function}

Having confirmed the excellent properties of the improved PT, we turn to focus on the baryon acoustic peak in the two-point correlation function. The two-point correlation function can be computed from the power spectrum as

$$
\xi(r)=\int \frac{d k k^{2}}{2 \pi^{2}} P_{11}(k) \frac{\sin (k r)}{k r} .
$$

Top panel of Fig. 8 shows the two-point correlation functions around the baryon acoustic peak at different redshifts $z=0.5,1,2$ and 3 (from top to bottom) in the case adopting the wmap5 cosmological parameters. Also, lower panel plots the fractional differences between N-body and improved PT results, i.e., $\left[\xi_{\mathrm{N}-\text { body }}(r)-\xi_{\mathrm{PT}}(r)\right] / \xi_{\mathrm{PT}}(r)$.

After the correction of finite-mode sampling, the error-bars in N-body simulations are greatly reduced, and the deviation of the N-body results from linear theory predictions (depicted as dotted lines) is clearly seen. As decreasing the redshift, the baryon acoustic peaks become smeared and the position of the peak are slightly shifted to a smaller scale. These trends can be accurately described by the leading-order calculation of improved PT, and the agreement between N-body results and the predictions is excellent. The fractional error in amplitude is well within a few percent, except for a large separation beyond the location of baryon acoustic peak, where the accuracy of N-body results tends to be worsen due to the limited simulation boxsize. Note that the corrections coming from the higher-order Born approximation do not alter the behaviors at $r>30 h^{-1} \mathrm{Mpc}$, and their amplitudes are negligibly small compared to the error-bars of N-body simulations. Thus the leading-order prediction seems robust for describing the baryon acoustic peak.

It has been recently suggested by several authors that the smearing effect on baryon acoustic peak is mostly attributed to the random motion of mass distribution [56], and it is approximately described by the convolution of the Gaussian smoothing function (e.g., [35, 57]). In the language of improved PT, this effect corresponds to the disappearance of the memory of initial condition, which is encoded in the non-linear propagator. Strictly speaking, the asymptotic behavior of the non-linear propagator is not a Gaussian form in closure approximation, although the damping behavior manifestly exhibits in the approximate solution of non-linear propagator. Hence, the prediction for the two-point correlation function seems robust against the high- $k$ behavior of the non-linear propagator.

Finally, it should be noted that the standard PT prediction fails to converge the integral in Eq. (4.4), because of the high- $k$ behavior of the power spectrum. This is true even when including the higher-order correction of two-loop 


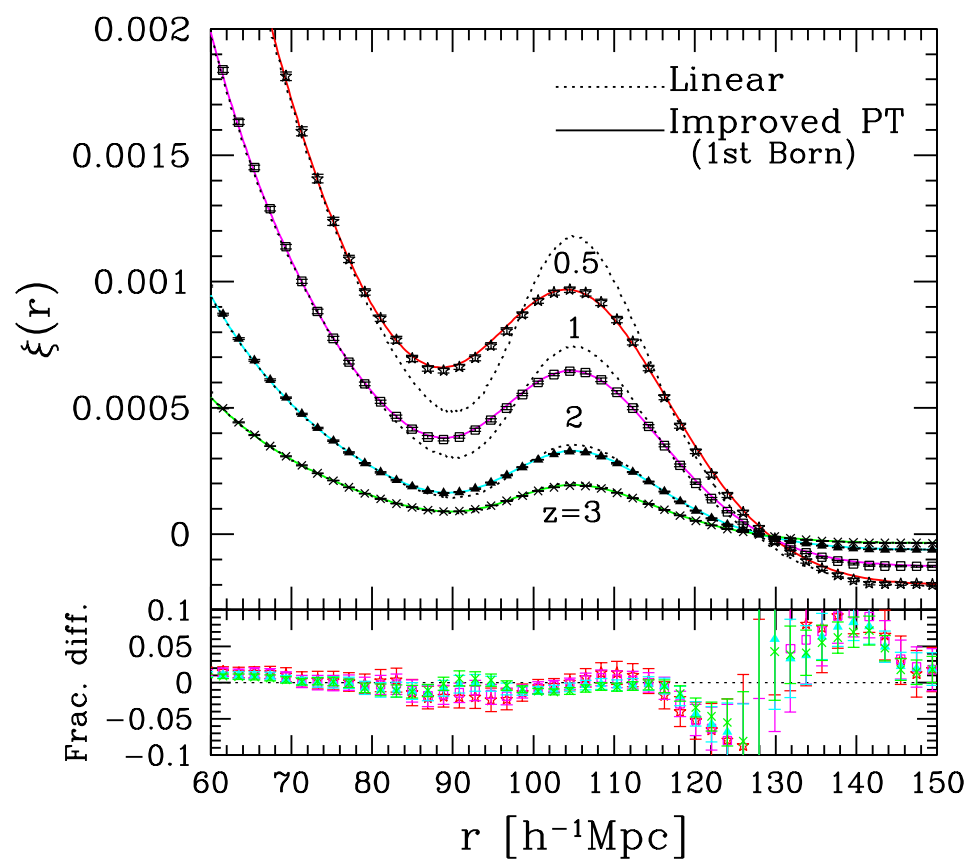

FIG. 8: Top: Two-point correlation functions in real space adopting the wmap5 cosmological parameters. The solid lines represent the leading-order predictions of improved PT, while the dotted lines show the linear theory results. Bottom: Fractional differences between N-body and improved PT results, $\left[\xi_{\mathrm{N}-\text { body }}(r)-\xi_{\mathrm{PT}}(r)\right] / \xi_{\mathrm{PT}}(r)$. In both panels, the symbols with error-bars indicate the N-body results averaged over the 30 realizations in which the effect of finite-mode sampling is corrected: $z=0.5$ (open stars), 1 (open squares), 2 (filled triangles), and 3 (crosses).

order. Thus, the successful results of improved PT prediction may be regarded as an outcome of non-perturbative property.

\section{Results in redshift space}

In practical observation with galaxy redshift surveys, the observed galaxy distribution is inevitably distorted due to the peculiar velocity of each galaxy. The so-called redshift-space distortion is known to alter the shape of the power spectrum in two different ways (e.g., [58]). One is the apparent enhancement of the clustering signal called Kaiser effect [59], which originates from the bulk motion of mass distribution falling into the massive halos. Another important effect is the finger-of-God (FoG) effect, which effectively suppresses the power spectrum amplitude on small scales by the virialized random motion of the mass residing at a halos.

Although a rigorous non-perturbative treatment of the redshift-space distortion is difficult, these two effects has been phenomenologically modeled as (e.g., 60, 61, 62, 63])

$$
P^{(\mathrm{S})}(k, \mu)=\left(1+\mu^{2} f\right)^{2} P_{11}(k) D_{\mathrm{FoG}}(k \mu),
$$

where $\mu$ is the cosine of the angle between the line-of-sight direction and the Fourier mode $\boldsymbol{k}$, and $f$ is the logarithmic derivative of linear growth factor, defined as $f \equiv d \ln D / d \ln a$. The function $D_{\text {FoG }}$ represents the damping function which mimics the FoG effect, and it asymptotically approaches unity in the $k \rightarrow 0$ limit, where the linear-theory formula by Kaiser is recovered.

Recently, Scoccimarro 64] proposed an improved version of the model (4.5) to properly take account of the nonlinear evolution of density and velocity fields on the Kaiser effect (see also [65, 66]). This is expressed as

$$
P^{(\mathrm{S})}(k, \mu)=\left[P_{11}(k)+2 f \mu^{2} P_{12}(k)+f^{2} \mu^{4} P_{22}(k)\right] \exp \left\{-\left(f \mu k \sigma_{\mathrm{v}}\right)^{2}\right\} .
$$



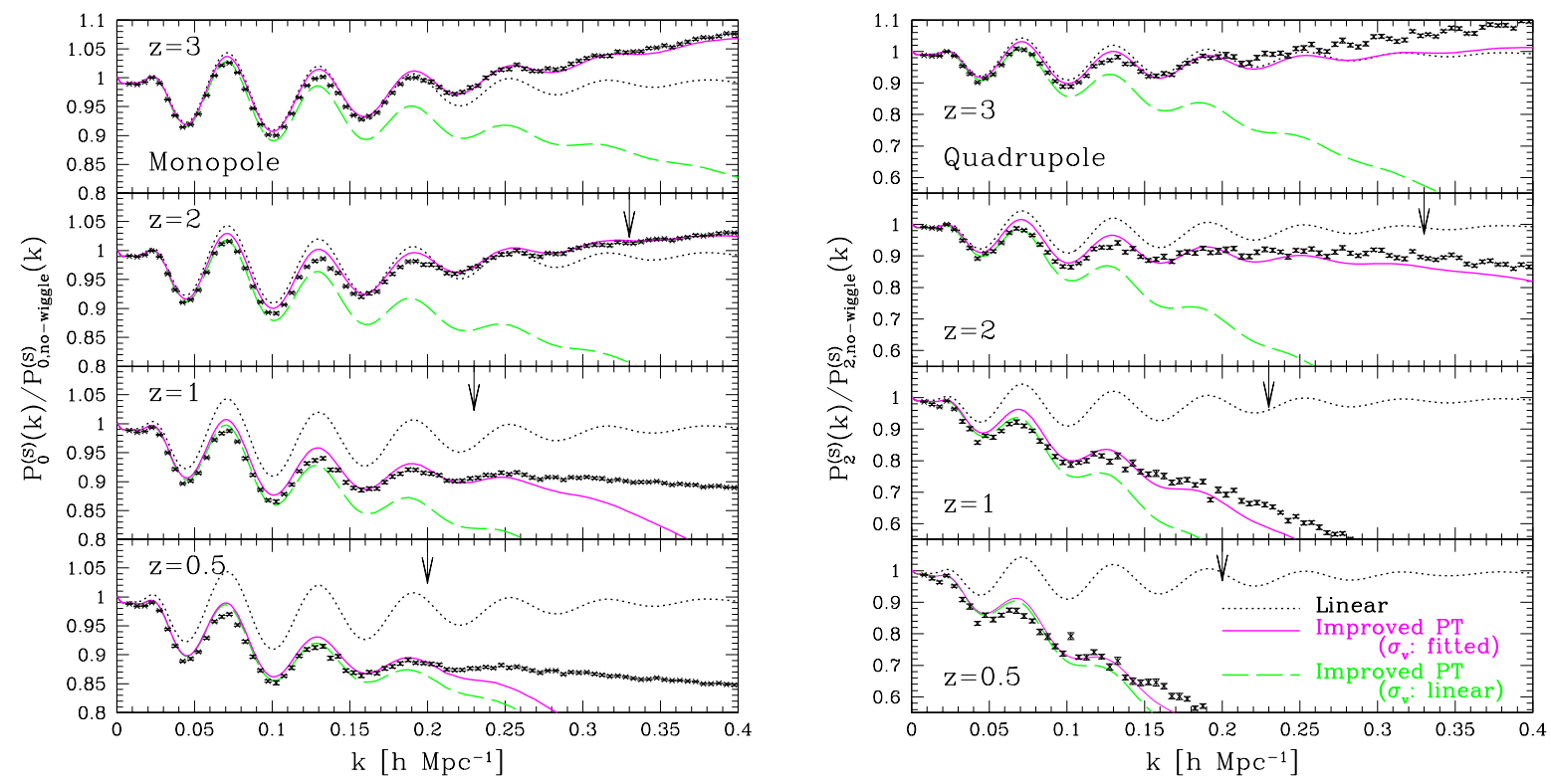

FIG. 9: Ratio of power spectra to smoothed reference spectra in redshift space, $P_{\ell}^{(\mathrm{S})}(k) / P_{\ell, \text { no-wiggle }}^{(\mathrm{S})}(k)$, from the wmap5 simulations. The reference spectrum $P_{\ell, \text { no-wiggle }}^{(\mathrm{S})}$ is calculated from the no-wiggle approximation of the linear transfer function, and the linear theory of the Kaiser effect is taken into account. Left panel shows the monopole power spectra $(\ell=0)$, and the right panel shows the quadrupole spectra $(\ell=2)$. Solid and dashed lines represent the results from the improved PT adopting the model of redshift-space distortion (4.6). To plot the results, the linear theory was used to compute $\sigma_{\mathrm{v}}$ in dashed lines, while in solid lines, $\sigma_{\mathrm{v}}$ was determined by fitting the predictions to the N-body simulations. In each panel, vertical arrow indicates the maximum wavenumber $k_{1 \%}$ for improved PT prediction including up to the second-order Born approximation, which has been estimated from Fig. 7 (see Sec. IVB 1 for definition of $k_{1 \%}$ ).

Here, the quantity $\sigma_{\mathrm{v}}$ is the one-dimensional velocity dispersion given by

$$
\sigma_{\mathrm{v}}^{2}=\frac{1}{3} \int \frac{d^{3} \boldsymbol{q}}{(2 \pi)^{3}} \frac{P_{22}(q)}{q^{2}} .
$$

In what follows, we adopt the model (4.6) to calculate the redshift-space power spectrum. Although this model is still phenomenological and may not be regarded as the best one, a comparison between the model predictions and N-body simulations shows that the prediction based on the model (4.6) gives a better result. Taking Eq. (4.6) as a canonical model of the redshift-space distortion, we will investigate the extent to which the model (4.6) faithfully reproduces the N-body results well, and discuss how to improve the model prescription.

\section{Power spectrum}

For a quantitative comparison of model prediction with N-body simulation, we compute the multipole moments of the two-dimensional power spectrum $P^{(\mathrm{S})}(k, \mu)$ :

$$
P_{\ell}^{(\mathrm{S})}(k)=\frac{2 \ell+1}{2} \int_{-1}^{1} d \mu P^{(\mathrm{S})}(k, \mu) \mathcal{P}_{\ell}(\mu)
$$

with $\mathcal{P}_{\ell}$ being the Legendre polynomials.

Substituting the model (4.6) into the above, the monopole, quadrupole and hexadecapole contribution to the redshift-space power spectrum are analytically expressed as

$$
\begin{aligned}
P_{0}^{(\mathrm{S})}(k) & =p_{0}(k), \\
P_{2}^{(\mathrm{S})}(k) & =\frac{5}{2}\left\{3 p_{1}(k)-p_{0}(k)\right\}, \\
P_{4}^{(\mathrm{S})}(k) & =\frac{9}{8}\left\{35 p_{2}(k)-30 p_{1}(k)+3 p_{0}(k)\right\} .
\end{aligned}
$$




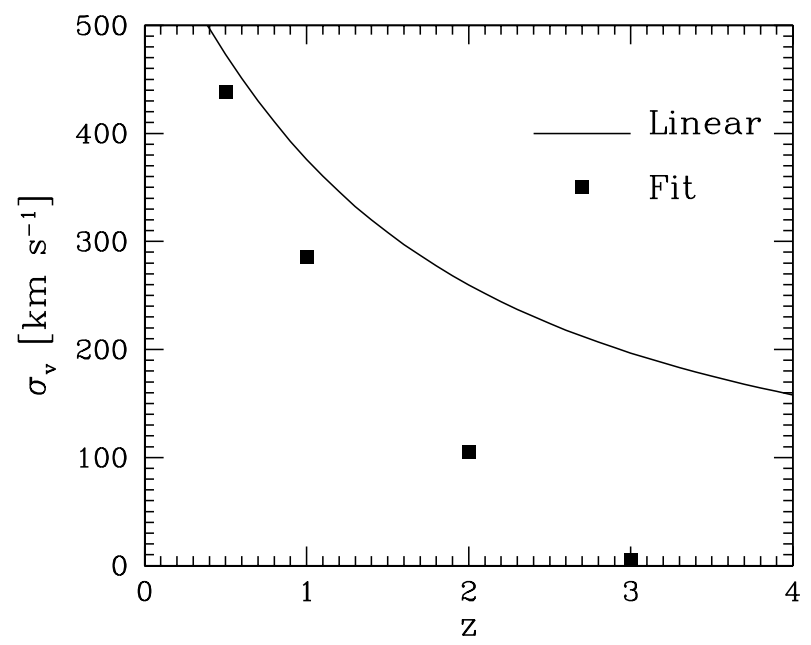

FIG. 10: Redshift evolution of velocity dispersion $\sigma_{\mathrm{v}}$. While the solid lines represent the linear theory prediction, the open squares indicate the results obtained by fitting the model (4.6) to the monopole and quadrupole spectra of N-body simulations (see Fig. 9).

where the function $p_{n}(k)$ is defined by

$$
p_{n}(k)=\frac{1}{2}\left[\frac{\gamma(n+1 / 2, \kappa)}{\kappa^{n+1 / 2}} P_{11}(k)+2 \frac{\gamma(n+3 / 2, \kappa)}{\kappa^{n+3 / 2}} f P_{12}(k)+\frac{\gamma(n+5 / 2, \kappa)}{\kappa^{n+5 / 2}} f^{2} P_{22}(k)\right] .
$$

The quantity $\gamma(n, \kappa)$ is the incomplete gamma function of the first kind

$$
\gamma(n, \kappa)=\int_{0}^{\kappa} d t t^{n-1} e^{-t}
$$

with its argument $\kappa=\left(k f \sigma_{\mathrm{v}}\right)^{2}$.

Fig. 9 shows the monopole (left) and quadrupole (right) moments of the redshift-space power spectra at different redshifts, obtained from the wmap5 simulations. We do not plot here the hexadecapole contributions, because the power spectrum estimated from the N-body simulations is still noisy even with the 30 realizations. In each panel of Fig. 9, the dashed lines indicate the improved PT predictions based on the model (4.6), where the corrections up to the second-order Born approximations are included. Note that the velocity dispersion $\sigma_{\mathrm{v}}$ is computed from the linear theory. Clearly, the predictions all underestimate the N-body results, and the agreement between predictions and $\mathrm{N}$-body simulations is restricted to a quite narrow range on large scales. As a reference, we also show the maximum wavenumber $k_{1 \%}$ of the improved PT prediction (vertical arrows), in which we include the corrections up to the second-order Born approximation in real space (see Fig. 7).

The reason why the prediction generically underestimates the N-body simulations would be partly attributed to the calculation of the velocity dispersion $\sigma_{\mathrm{v}}$ using the linear theory. It has been advocated by several authors that the suppression of power spectrum by FoG effect is originated from the non-linear structure of virialized halos, and thereby the linear theory estimation of $\sigma_{\mathrm{v}}$ may be inappropriate. In this respect, we admittedly regard $\sigma_{\mathrm{v}}$ as an uncontrollable parameter, which should be determined by fitting the predictions to N-body results.

The solid lines in each panel of Fig. 9 show the results of redshift-space spectra adopting the fitted values of $\sigma_{\mathrm{v}}$. In estimating $\sigma_{\mathrm{v}}$, both the monopole and quadrupole spectra were fitted to the N-body results in the range of $0 \leq k \leq k_{1 \%}$. Fig. 10 summarizes the fitted results of $\sigma_{\mathrm{v}}$, which significantly deviate from the linear theory prediction at higher redshifts.

Then, apparently, overall agreement between prediction and simulation becomes fairly improved, although as a trade-off, small discrepancy manifests at low-k mode, where the N-body results rather agree well with the prediction adopting $\sigma_{\mathrm{v}}$ calculated from linear theory. In Fig. 11, left and right panels respectively plot the fractional differences of the monopole and quadrupole moments between the model predictions and N-body simulations. Except for the narrow range of low-k modes, a percent-level agreement is almost achieved for the monopole power spectrum. This is true at least within the convergence regime calibrated in real space (see vertical arrows in Fig. 11). However, the 

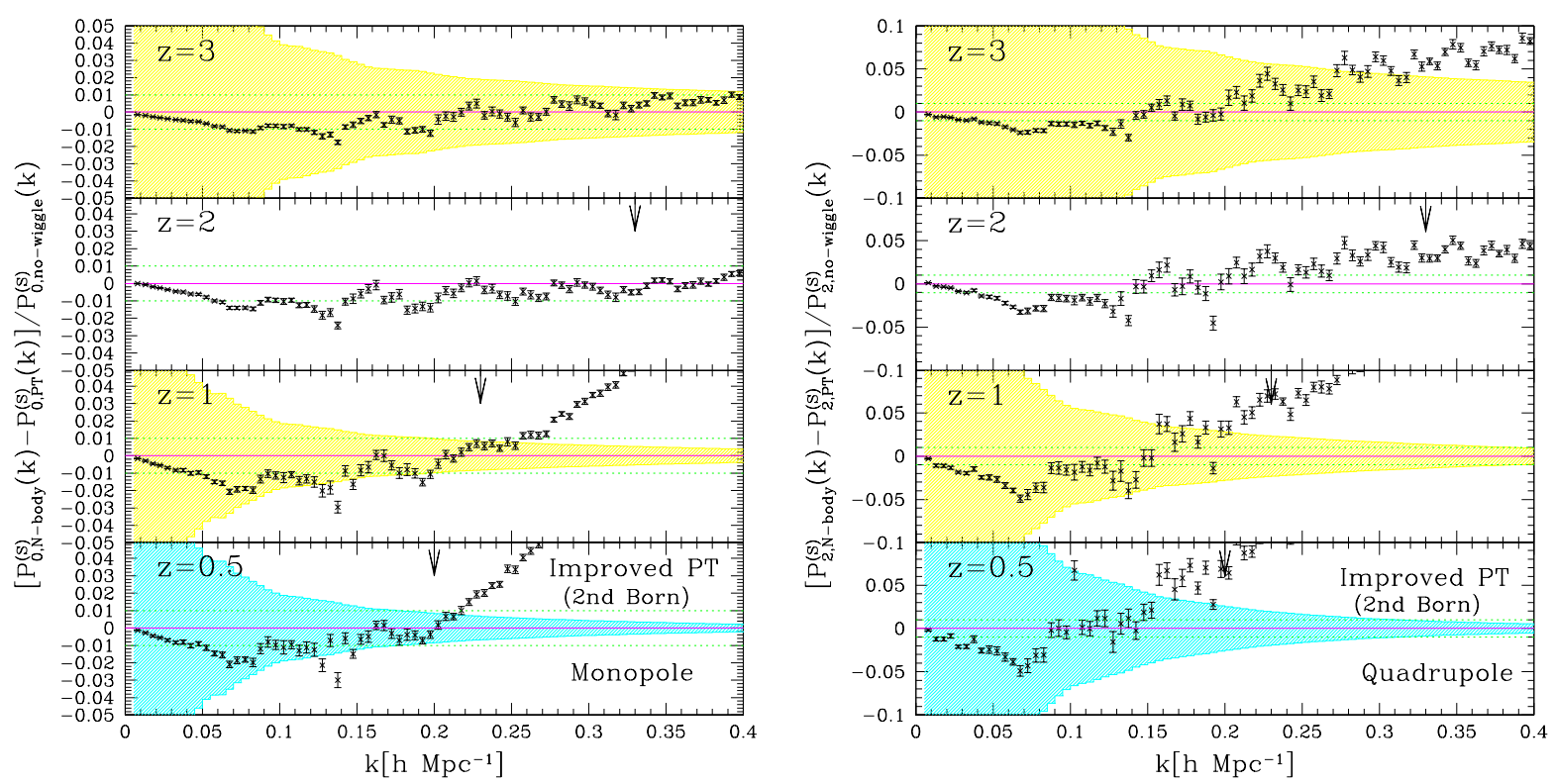

FIG. 11: Difference between N-body and PT results divided by the reference spectrum in redshift space, i.e., $\left[P_{\ell, \mathrm{N}-\text { body }}^{(\mathrm{S})}(k)-\right.$ $\left.P_{\ell, \mathrm{PT}}^{(\mathrm{S})}(k)\right] / P_{\ell, \text { no-wiggle }}^{(\mathrm{S})}(k)$. The left and right panels respectively represent the results from monopole and quadrupole power spectra. Note that the improved PT predictions are computed based on the model (4.6) adopting the fitted value of $\sigma_{\mathrm{v}}$. For comparison, the statistical errors limited by the cosmic variance of the survey volumes roughly corresponding to those of WFMOS-like survey [28] and BOSS 29] are shown as shaded regions in panels of $z=3, z=1$ and $z=0.5$, assuming respectively the survey volumes of $V=1 h^{-3} \mathrm{Gpc}^{3}, 4 h^{-3} \mathrm{Gpc}^{3}$ and $4.5 h^{-3} \mathrm{Gpc}^{3}$. Note that in each panel, vertical arrow indicates the maximum wavenumber $k_{1 \%}$ determined from Fig. 7 by comparison between N-body and improved PT results.

fractional error of the quadrupole power spectrum still exhibits a little bit large discrepancy, signaling the fact that the model (4.6) misses something important for higher-multipole moment of redshift-space distortion.

To see the significance of this deviation in practice, in Fig. 11, the expected 1- $\sigma$ errors limited by the cosmic variance, $\Delta P_{\ell}^{(\mathrm{S})}(k)$, are shown, depicted as the shaded region. Here, we specifically consider the ground-based BAO surveys like WFMOS survey [28] and BOSS 29], assuming the survey volumes of $V=1 h^{-3} \mathrm{Gpc}^{3}$ at $z=3$ and $4 h^{-3} \mathrm{Gpc}^{3}$ at $z=1$ for WFMOS survey, and $V=4.5 h^{-3} \mathrm{Gpc}^{3}$ at $z=0.5$ for BOSS ${ }^{5}$. Based on the approximation that the density field is well-described by a Gaussian random field, the cosmic-variance error $\Delta P_{\ell}^{(\mathrm{S})}(k)$ can be estimated as

$$
\left[\Delta P_{\ell}^{(\mathrm{S})}(k)\right]^{2}=\frac{2}{N_{k}} \sigma_{P, \ell}^{2}(k),
$$

where the quantity $N_{k}$ is the number of Fourier modes within a given bin at $k$, and is given by $N_{k}=$ $4 \pi k^{2} \Delta k /\left(2 \pi / L_{\mathrm{box}}\right)^{3} / 2=V k^{2} \Delta k /(2 \pi)^{2}$. The function $\sigma_{P, \ell}$ is

$$
\sigma_{P, \ell}^{2}(k)=\frac{(2 \ell+1)^{2}}{2} \int_{-1}^{1} d \mu\left\{P^{(\mathrm{S})}(k, \mu) \mathcal{P}_{\ell}(\mu)\right\}^{2} .
$$

The expression (4.14) with (4.15) is a generalization of the cosmic-variance error in real space (e.g., [47, 67, 68, 69]) to the multipole moments in redshift space. Note that the error $\Delta P_{\ell}^{(\mathrm{S})}(k)$ depends on the bin width $\Delta k$, for which we simply adopt the same bin size as used in the power spectrum analysis of N-body data. The analytic estimate of $\Delta P_{\ell}^{(\mathrm{S})}$ based on Eq. (4.14) is roughly consistent with the statistical errors estimated from the N-body data of 30 realizations.

Comparison between the cosmic-variance errors and fractional differences shows that the discrepancy seen in the quadrupole power spectrum is definitely large, and it eventually exceeds the statistical error at large $k$ modes. Since

\footnotetext{
${ }^{5}$ Strictly speaking, BOSS project is a part of Sloan Digital Sky Survey III, aiming at precisely measuring the cosmological distance and expansion rate at $z=0.35,0.6$ and $z=2.5$. Here, we only consider the low- $z$ measurement with survey depth $0.2 \lesssim z \lesssim 0.8$.
} 
this has happened inside the valid range of the improved PT calibrated in real space (indicated as vertical arrows), we conclude that the current model prediction with (4.6) is insufficient to describe the higher-multipole moments of BAOs, and a more elaborate work on the models of redshift-space distortion is needed for upcoming BAO measurement.

\section{Correlation function}

Finally, we discuss the correlation functions in redshift space. Similar to the power spectrum, we apply the multipole expansion to the anisotropic two-point correlation function as

$$
\xi^{(\mathrm{S})}\left(s_{\|}, s_{\perp}\right)=\int \frac{d^{3} \boldsymbol{k}}{(2 \pi)^{3}} P^{(\mathrm{S})}(k, \mu) e^{i \boldsymbol{k} \cdot \boldsymbol{s}}=\sum_{\ell: \text { even }} \xi_{\ell}^{(\mathrm{S})}(s) \mathcal{P}_{\ell}(\nu)
$$

with $\nu=s_{\|} / s$. The multipole moment of the correlation function, $\xi_{\ell}^{(\mathrm{S})}$, is directly related to the Fourier counterpart, $P_{\ell}^{(\mathrm{S})}$ through

$$
\xi_{\ell}^{(\mathrm{S})}(s)=i^{\ell} \int \frac{d k k^{2}}{2 \pi^{2}} P_{\ell}^{(\mathrm{S})}(k) j_{\ell}(k s) .
$$

Fig. 12 shows the monopole (left), quadrupole (middle) and hexadecapole (right) moments of correlation function. In each panel, the N-body results are compared with the predictions from linear theory (dotted) and the leadingorder calculation of improved PT (solid) adopting the model (4.6) with linear theory prediction of $\sigma_{\mathrm{v}}$. Note that the predictions of improved PT are hardly changed by including the higher-order corrections and/or using the fitted value of $\sigma_{\mathrm{v}}$, at least around the baryon acoustic peak, and the systematic differences between including and ignoring the corrections are well within the error-bars of N-body simulations.

As anticipated from the results in real space, the baryon acoustic peaks in the monopole moment tend to be smeared as decreasing redshift, but the effect seems little bit stronger than those in real space. This is due to the additional effect coming from the redshift distortion. Although no prominent signal of the BAOs exists in the highermultipole moments, the same tendencies can be seen in the quadrupole and hexadecapole moments. The improved PT calculations are broadly consistent with N-body results, but small discrepancies manifest around the baryon acoustic peak and trough. Lower panels of Fig. 12 showing the fractional differences imply that these are at most $5 \%$ effect in amplitude, except for the hexadecapole case with large error-bars of simulation. It is interesting to note that no noticeable redshift dependence appears in the fractional differences, indicating that the discrepancies may be attributed to the model of redshift-space distortion. Furthermore, it turns out that these are well within the cosmicvariance errors of the ground-based BAO measurement, indicated as shaded region. Assuming that the underlying density field is well described by a Gaussian random field, the cosmic variance for the multipole correlation functions $\xi_{\ell}^{(\mathrm{S})}$ can be written as (see [57, 70, 71] for cosmic-variance errors in real space)

$$
\left[\Delta \xi_{\ell}^{(\mathrm{S})}(s)\right]^{2}=\frac{2}{V} \int \frac{d k k^{2}}{2 \pi^{2}}\left\{j_{\ell}(k s) \sigma_{P, \ell}(k)\right\}^{2}
$$

with $\sigma_{P, \ell}$ given by Eq. (4.15). Note that the analytic estimation of cosmic-variance errors $\Delta \xi_{\ell}^{(\mathrm{S})}$ shown in Fig. 12 reproduce the N-body results quite well.

Hence, compared to the power spectrum in redshift space, the correlation functions obtained from the N-body simulation and analytic calculation can have a better agreement. Presumably, this is because the acoustic peak structure in the correlation function is mostly attributed to the low-k behavior of the BAOs, and the power spectrum at low-k modes is accurately described by the model (4.6) with the linear theory prediction of $\sigma_{\mathrm{v}}$. In other words, the baryon acoustic peak would be robust against the non-linear effects at high-k modes (see also [35, 36, 72]). This implies that even the prediction at the current level is sufficient to characterize the acoustic peak in the correlation function, and it can be used as an accurate theoretical template for future precision BAO measurement.

Note, however, that the measured amplitudes of the two-point correlation function are strongly correlated between different scales. In practice, not only the diagonal component but also the off-diagonal components of the covariance of the correlation function must be considered for a reliable estimation of cosmological distance, and a more careful study is needed.

\section{DISCUSSION AND CONCLUSIONS}

In this paper, we have presented the improved PT calculations of the matter power spectrum and two-point correlation function in real and redshift spaces. Based on the closure approximation of the renormalized PT treatment, 

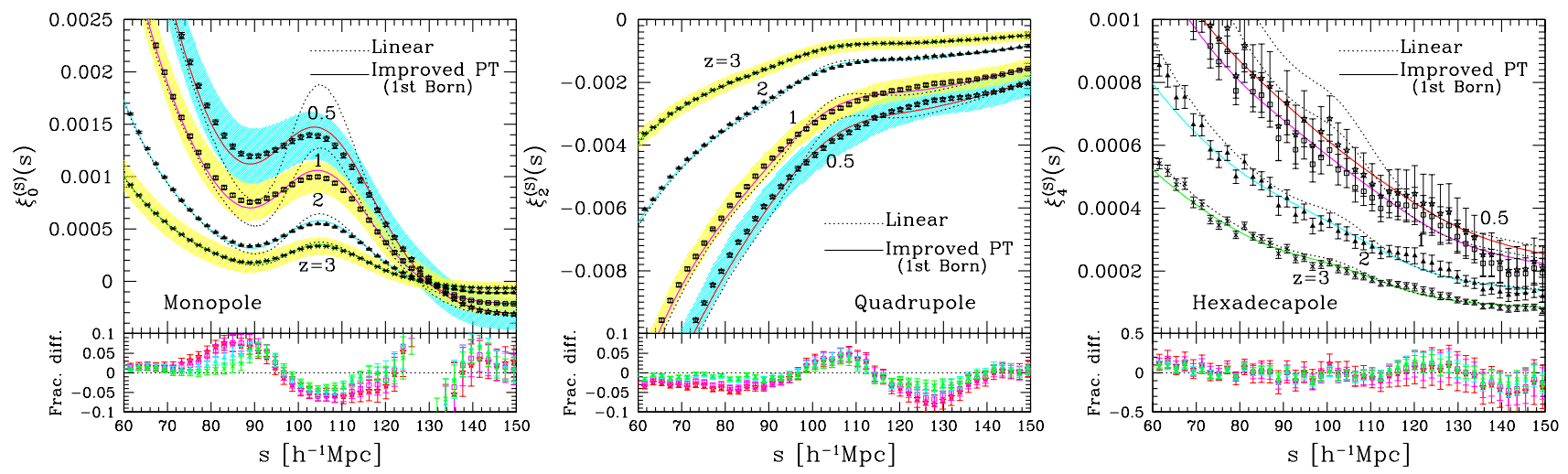

FIG. 12: Top: Correlation function in redshift space. Left, middle and right panel respectively shows the monopole, quadrupole and hexadecapole contributions to the anisotropic correlation function $\xi^{(\mathrm{S})}$. The solid and dotted lines are the predictions from the improved PT based on the model (4.6) and linear theory, respectively. Note that only the leading-order Born approximation to the the mode coupling term is included in the improved PT. $z=0.5$ (red); $z=1$ (magenta); $z=2$ (cyan); $z=3$ (green). For comparison, the statistical errors limited by the cosmic variance of the survey volumes $V=1 h^{-3} \mathrm{Gpc}^{3}, 4 h^{-3} \mathrm{Gpc}^{3}$ and $4.5 h^{-3} \mathrm{Gpc}^{3}$ are estimated from Eq. (4.18), and are depicted as shaded regions around the N-body results at $z=3, z=1$ and $z=0.5$, respectively. The cosmic-variance error for hexadecapole is not shown here because of the large scatter. Bottom: Fractional differences of the results between N-body simulations and improved PT predictions, $\left[\xi_{\mathrm{N}-\text { body }}(s)-\xi_{\mathrm{PT}}(s)\right] / \xi_{\mathrm{PT}}(s)$ for different redshifts at $z=0.5$ (open stars), $z=1$ (open squares), $z=2$ (filled triangles), and $z=3$ (crosses).

a closed set of the non-perturbative expressions for power spectrum and propagator is obtained. The resultant expression includes the effect of resummation for a class of loop diagrams at infinite order, and thereby the convergence of higher-order contributions is expected to be improved. Employing the Born approximation, we have analytically calculated the non-linear power spectrum, and compared the convergence properties of improved PT with those of standard PT by explicitly computing the higher-order corrections.

We have also made a detailed comparison between the improved PT result and N-body simulations. With a large boxsize and many realization data of N-body simulations, the statistical errors of two-point statistics are greatly reduced by the correction of the effect of finite-mode sampling, and this enables us to investigate the convergence check of numerical and analytic calculations at a percent level. Then, specifically focusing on the behaviors of BAOs, the power spectrum and two-point correlation functions are calculated in both real and redshift spaces. In redshift space, the effect of redshift-space distortion which changes the clustering pattern of mass distribution should be incorporated into the improved PT predictions. In this paper, adopting the model proposed by Ref. [64] (Eq. (4.6) ), we have quantified the extent to which the current model description faithfully reproduces the N-body results, and clarified the key ingredients toward an improved prescription of redshift-space distortion.

Our important findings are summarized as follows:

- The improved PT expansion based on the Born approximation has better convergence properties, in marked contrast with the standard PT expansion. The corrections coming from the mode-coupling term are welllocalized positive functions of wavenumbers, and their contributions tend to be shifted to a higher $k$ region as increasing the order of perturbation. Thus, the inclusion of higher-order corrections stably improves the prediction, and the range of agreement with N-body results becomes wider in wavenumber.

- In real-space power spectrum, the improved PT prediction including up to the second-order Born correction seems essential for modeling BAO precisely. We estimated the maximum wavenumber $k_{1 \%}$, below which the results of both the N-body simulation and improved PT calculation converge well within the $1 \%$ accuracy. The resultant value of $k_{1 \%}$ can be summarized as Eq. (4.3) with the constant value $C=0.7$, which provides a way to estimate $k_{1 \%}$ in a cosmology independent manner. On the other hand, if we consider the two-point correlation function in real space, the leading-order calculation turns out to be sufficiently accurate, and no higher-order correction is needed to describe the non-linear evolution of baryon acoustic peak seen in the N-body simulations.

- Modeling redshift-space power spectrum with Eq. (4.6) gives a broadly consistent result with N-body simulations, if we regard the velocity dispersion $\sigma_{\mathrm{v}}$ as a fitting parameter. However, discrepancy between improved PT predictions and N-body results has appeared in the quadrupole power spectrum, and it becomes larger than the 
statistical errors limited by the cosmic variance of the survey volume $V \sim$ a few $h^{-3} \mathrm{Gpc}^{3}$. This is true even in the valid range of improved $\mathrm{PT}, k \lesssim k_{1 \%}$. On the other hand, while a small descrepancy has been also found in the two-point correlation, it turns out that the discrepancy is well within the cosmic-variance error, and even the leading-order prediction using the linear theory estimate of $\sigma_{\mathrm{v}}$ can be used as an accurate theoretical template for future ground-based $\mathrm{BAO}$ measurement.

The recently proposed techniques to deal with the non-linear gravitational clustering, including the present treatment, have been greatly developed, and they would be a promising cosmological tool to precisely model the shape and amplitude of the power spectrum and/or the correlation functions in an accuracy of sub-percent level. Combining the model of redshift-space distortion, we are now able to discuss the non-linear clustering in redshift space. Although the present paper is especially concerned with the analytical work, we note that the non-perturbative formulation with closure approximation is suited for forward treatment in time [48], in which all orders of Born approximation can be fully incorporated into the predictions by numerically solving the evolution equations. This approach would be particularly useful to study the non-linear matter power spectrum in general cosmological models, including the modified theory of gravity [49].

Finally, in practical application to the precision BAO measurements, there are several remaining issues to be addressed in the future work. The improvement of the model of redshift-space distortion is, of course, a very important and urgent task. The effect of galaxy biasing is also one of the key ingredients for modeling accurate theoretical template, and several attempts to take account of this effect have been recently made [36, 73, 74, 75, 76, 77, 78]. Another interesting direction is to develop a fast computation of non-linear power spectrum or correlation function for an arbitrary cosmological model. Recently, the statistical sampling method for precise power spectrum emulation has been proposed [79, 80, 81]. In this treatment, only a limited set of cosmological models can be used to predict power spectrum at the required accuracy over the prior parameter ranges. The analytic approaches combining this method may provide a fast and reliable way to estimate the two-point statistics, and the development of this method would be valuable.

\section{Acknowledgments}

We would like to thank Yasushi Suto and Alan Heavens for comments and discussion, Thierry Sousbie for teaching us an efficient computational method for two-point correlation function. AT is supported by a Grant-in-Aid for Scientific Research from the Japan Society for the Promotion of Science (JSPS) (No. 21740168). TN and SS acknowledge a support from JSPS fellows. This work was supported in part by Grant-in-Aid for Scientific Research on Priority Areas No. 467 "Probing the Dark Energy through an Extremely Wide and Deep Survey with Subaru Telescope", and JSPS Core-to-Core Program "International Research Network for Dark Energy".

\section{APPENDIX A: STANDARD PERTURBATION THEORY UP TO THE TWO-LOOP ORDER}

In this Appendix, we briefly summarize the standard PT and derive a set of perturbative solutions. Based on these solutions, we obtain the analytic expressions for power spectrum up to the two-loop order.

As we mentioned in Sec. IIIA, standard PT is the straightforward expansion of the quantity $\Phi_{a}$, and the perturbative solutions are obtained by order-by-order treatment of Eq. (2.2). In order to systematically derive the solutions, the Einstein-de Sitter (EdS) approximation is often used in the literature. In the EdS approximation, the matrix $\Omega_{a b}$ given by Eq. (2.3) is replaced with the one in the EdS universe, i.e., $\Omega(\eta)=1$ and $f=d \ln D / d \ln a=1$. This means that all the non-linear growth factors appearing in the higher-order solutions are expressed in terms of the linear growth factor $D(t)$. Neglecting the contributions from the decaying mode, the resultant solution for $\Phi_{a}$ is then expanded as

$$
\Phi_{a}(\boldsymbol{k} ; \eta)=e^{\eta} \Phi_{a}^{(1)}(\boldsymbol{k} ; \eta)+e^{2 \eta} \Phi_{a}^{(2)}(\boldsymbol{k} ; \eta)+e^{3 \eta} \Phi_{a}^{(3)}(\boldsymbol{k} ; \eta)+\cdots,
$$

The solution for each order of perturbation is expressed as

$$
\Phi_{a}^{(n)}(\boldsymbol{k})=\int \frac{d^{3} \boldsymbol{k}_{1} \cdots d^{3} \boldsymbol{k}_{n}}{(2 \pi)^{3(n-1)}} \delta_{\mathrm{D}}\left(\boldsymbol{k}-\boldsymbol{k}_{1}-\cdots-\boldsymbol{k}_{n}\right) \mathcal{F}_{a}^{(n)}\left(\boldsymbol{k}_{1}, \cdots, \boldsymbol{k}_{n}\right) \delta_{0}\left(\boldsymbol{k}_{1}\right) \cdots \delta_{0}\left(\boldsymbol{k}_{n}\right),
$$

where $\delta_{0}$ is the initial density field which we assume Gaussian statistic. The function $\mathcal{F}_{a}^{n}$ is the symmetrized kernel of the $n$-th order solutions. The explicit expressions for the kernel $F_{a}^{(n)}$ is obtained from the recursion relation, which 
can be derived by substituting the expansion (A1) with (스) into Eq. (2.2) (e.g., [32, 47, 82, 83]):

$$
\begin{aligned}
& F_{a}^{(1)}\left(\boldsymbol{k}_{1}\right)=(1,1), \\
& F_{a}^{(n)}\left(\boldsymbol{k}_{1}, \cdots, \boldsymbol{k}_{n}\right)=\sigma_{a b}^{(n)} \sum_{m=1}^{n-1} \gamma_{b c d}\left(\boldsymbol{q}_{1}, \boldsymbol{q}_{2}\right) F_{c}^{(m)}\left(\boldsymbol{k}_{1}, \cdots, \boldsymbol{k}_{m}\right) F_{d}^{(n-m)}\left(\boldsymbol{k}_{n-m+1}, \cdots, \boldsymbol{k}_{n}\right)
\end{aligned}
$$

with $\boldsymbol{q}_{1} \equiv \boldsymbol{k}_{1}+\cdots+\boldsymbol{k}_{m}$ and $\boldsymbol{q}_{2} \equiv \boldsymbol{k}_{m+1}+\cdots+\boldsymbol{k}_{n}$. Here, the matrix $\sigma_{a b}^{(n)}$ is given by

$$
\sigma_{a b}^{(n)}=\frac{1}{(2 n+3)(n-1)}\left(\begin{array}{cc}
2 n+1 & 2 \\
3 & 2 n
\end{array}\right) .
$$

Note that the kernel $F_{a}^{(n)}$ given above is not yet symmetric under the permutations of arguments, $\boldsymbol{k}_{1}, \cdots, \boldsymbol{k}_{n}$, and it should be symmetrized:

$$
\mathcal{F}_{a}^{(n)}=\frac{1}{n !} \sum_{\text {permutations }} F_{a}^{(n)}\left(\boldsymbol{k}_{1}, \cdots, \boldsymbol{k}_{n}\right)
$$

Using the perturbative solutions, the power spectrum defined by (3.1) is expanded as

$$
P_{a b}(k ; \eta)=e^{2 \eta} P_{a b}^{(11)}(k)+e^{4 \eta}\left\{P_{a b}^{(22)}(k)+P_{a b}^{(13)}(k)\right\}+e^{6 \eta}\left\{P_{a b}^{(33)}(k)+P_{a b}^{(24)}(k)+P_{a b}^{(15)}(k)\right\}+\cdots .
$$

Here, the quantity $P^{(m n)}$ implies the ensemble average obtained from the $m$-th and $n$-th order perturbative solutions. In the above expression, the first term at the right-hand side is the linear power spectrum, while the second and third terms proportional to the growth factors $e^{4 \eta}$ and $e^{6 \eta}$ are respectively the so-called one-loop and two-loop corrections. The explicit expressions for these corrections become (e.g., [46, 84])

$$
\begin{aligned}
P_{a b}^{(11)}(k)= & u_{a} u_{b} P_{0}(k) \\
P_{a b}^{(22)}(k)= & 2 \int \frac{d^{3} \boldsymbol{q}}{(2 \pi)^{3}} \mathcal{F}_{a}^{(2)}(\boldsymbol{q}, \boldsymbol{k}-\boldsymbol{q}) \mathcal{F}_{b}^{(2)}(\boldsymbol{q}, \boldsymbol{k}-\boldsymbol{q}) P_{0}(q) P_{0}(|\boldsymbol{k}-\boldsymbol{q}|) \\
P_{a b}^{(13)}(k)= & 3 P_{0}(k) \int \frac{d^{3} \boldsymbol{q}}{(2 \pi)^{3}}\left\{\mathcal{F}_{a}^{(3)}(\boldsymbol{k}, \boldsymbol{q},-\boldsymbol{q})+\mathcal{F}_{b}^{(3)}(\boldsymbol{k}, \boldsymbol{q},-\boldsymbol{q})\right\} P_{0}(q) \\
P_{a b}^{(33)}(k)= & 9 P_{0}(k) \int \frac{d^{3} \boldsymbol{p} d^{3} \boldsymbol{q}}{(2 \pi)^{6}} \mathcal{F}_{a}^{(3)}(\boldsymbol{k}, \boldsymbol{p},-\boldsymbol{p}) \mathcal{F}_{b}^{(3)}(\boldsymbol{k}, \boldsymbol{q},-\boldsymbol{q}) P_{0}(p) P_{0}(q) \\
& +6 \int \frac{d^{3} \boldsymbol{p} d^{3} q}{(2 \pi)^{6}} \mathcal{F}_{a}^{(3)}(\boldsymbol{p}, \boldsymbol{q}, \boldsymbol{k}-\boldsymbol{p}-\boldsymbol{q}) \mathcal{F}_{b}^{(3)}(\boldsymbol{p}, \boldsymbol{q}, \boldsymbol{k}-\boldsymbol{p}-\boldsymbol{q}) P_{0}(p) P_{0}(q) P_{0}(|\boldsymbol{k}-\boldsymbol{p}-\boldsymbol{q}|) \\
P_{a b}^{(24)}(k)= & 12 \int \frac{d^{3} \boldsymbol{p} d^{3} \boldsymbol{q}}{(2 \pi)^{6}}\left\{\mathcal{F}_{a}^{(2)}(\boldsymbol{p}, \boldsymbol{k}-\boldsymbol{p}) \mathcal{F}_{b}^{(4)}(\boldsymbol{p}, \boldsymbol{q},-\boldsymbol{q}, \boldsymbol{k}-\boldsymbol{p})+\mathcal{F}_{a}^{(4)}(\boldsymbol{p}, \boldsymbol{q},-\boldsymbol{q}, \boldsymbol{k}-\boldsymbol{p}) \mathcal{F}_{b}^{(2)}(\boldsymbol{p}, \boldsymbol{k}-\boldsymbol{p})\right\} \\
& \times P_{0}(p) P_{0}(q) P_{0}(|\boldsymbol{k}-\boldsymbol{p}|) \\
P_{a b}^{(15)}(k)= & 15 P_{0}(k) \int \frac{d^{3} \boldsymbol{p} d^{3} \boldsymbol{q}}{(2 \pi)^{6}}\left\{\mathcal{F}_{a}^{(5)}(\boldsymbol{p}, \boldsymbol{q}, \boldsymbol{k},-\boldsymbol{p},-\boldsymbol{q})+\mathcal{F}_{b}^{(5)}(\boldsymbol{p}, \boldsymbol{q}, \boldsymbol{k},-\boldsymbol{p},-\boldsymbol{q})\right\} P_{0}(p) P_{0}(q),
\end{aligned}
$$

where $P_{0}$ is the initial power spectrum of the density field $\delta_{0}$ defined by Eq. (2.7), and we set $u_{a}=(1,1)$.

Note that the expression for one-loop power spectra can be further reduced to the one-dimensional and twodimensional integral for $P^{(13)}$ and $P^{(22)}$, respectively (e.g., 83, 85, 86, 87]). In the results presented in Sec. [IVB1, we used the method of Gaussian quadratures for numerical integration of one-loop power spectra. On the other hand, for the two-loop power spectra, the integration cannot be simplified except for the first term in $P^{(33)}$, and we need to directly evaluate the six-dimensional integration. We adopted the Monte-Carlo integration to the two-loop power spectra. The integration kernels for each term are generated numerically using the recursion relation (A4) and the condition (A5).

\section{APPENDIX B: COMPARISON TO OTHER WORKS}

In this Appendix, we collect several recent works that attempt to improve the prediction of power spectrum and/or two-point correlation function, and discuss their qualitative differences. A quantitative aspect of various analytic 


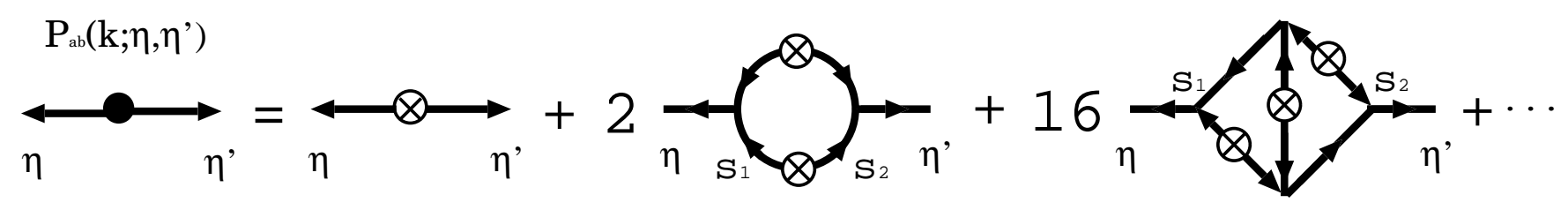

FIG. 13: Diagrammatic representation for the perturbative treatment of the power spectrum proposed by Crocce \& Scoccimarro (2008), based on the renormalized PT. This is compared with Fig. 3
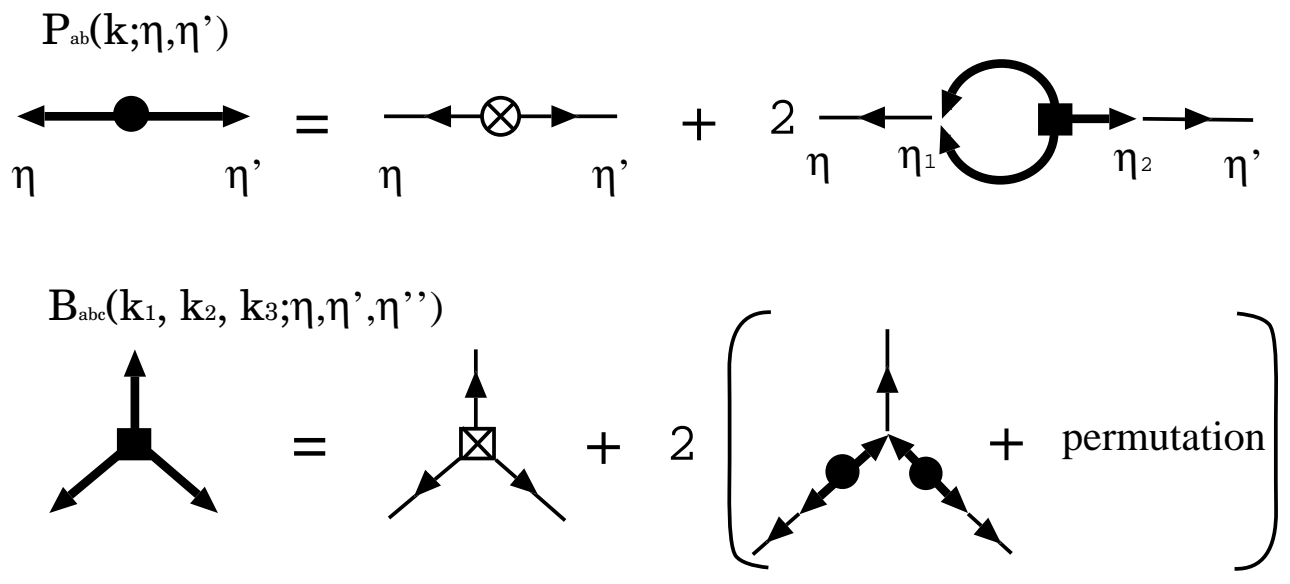

FIG. 14: Diagrammatic representation of the non-perturbative treatment proposed by Pietroni (2008), which can be compared with Fig. 2

methods has been recently investigated in Ref. [46]. Here, we specifically comment on the approaches proposed by Refs. [34, 35, 39, 42], which are very close to our treatment.

Crocce \& Scoccimarro (2008) : First let us mention the work by Ref. 34]. Although the treatment presented in the paper are often quoted as 'RPT', strictly speaking, this is just the approximate treatment, which differs from the renormalized PT [32]. As we mentioned in Sec. III A renormalized PT is the exact non-perturbative formulation without any approximations, and the power spectrum given by Eq. (3.4) is expressed as the infinite series of irreducible loop diagrams constructed from the non-linear propagator, full vertex, and non-linear power spectrum. To make the analysis tractable, they adopted the following approximations: (i) the renormalized vertex is well-described by the (linear) vertex function; (ii) the non-linear power spectra that enter into the calculation of $P_{a b}^{(\mathrm{MC})}$ are all replaced with the linear-order ones. In our language, this corresponds to the firstorder Born approximation. Then, using the approximate solution for propagator in Ref. [33], they explicitly calculated the power spectrum including the corrections up to the two-loop order. The diagrams that they actually computed are shown in Fig. 13.

Compared to our analytical treatment with Born approximation, there are two main differences. One is the higher-order corrections that appear in the diagrams (see Fig. 3). Another important difference is the asymptotic behaviors in the non-linear propagator. At $k \rightarrow \infty$, the propagator used in their paper behaves like $G_{a b} \rightarrow$ $g_{a b} \exp \left[-x^{2} / 2\right]$, which contrasts with $G_{a b} \rightarrow g_{a b} J_{1}(2 x) / x$ in our closure approximation, where $g_{a b}$ is the linear propagator and $x$ is defined by $x \equiv k \sigma_{\mathrm{v}}\left(e^{\eta}-e^{\eta^{\prime}}\right)$. These distinctive features come from the partial resummation of a different class of higher-order terms when constructing the approximate solution of non-linear propagator (see Ref. [33, 43] in details). Despite these remarkable differences, it has been shown in Ref. 45] that the leading-order calculations neglecting the higher-order terms (two-loop diagram or second-order Born correction) can produce the same results which is indistinguishable from each other. This is true at least on large scales, where the agreement between N-body simulations and improved PT predictions is better than a few percent.

Pietroni (2008) : Next consider the method proposed by Ref. [39], called time-RG method. This method is based on the moment-based approach, and we first write down the moment equations. In general, this produces an infinite hierarchy of equations, however, Ref. 39] assumes a vanishing trispectrum in order to truncate the hierarchy. As a result, a closed set of equations for power spectrum $P_{a b}$ is obtained, which coulples with the evolution of bispectrum $B_{a b c}$ in some non-perturbative ways. Diagrammatic representation of this closed equations is shown 
in Fig. 14, which can be compared with Fig. 2 in our treatment. Note that in the subject of statistical theory of turbulence, this truncation procedure is referred to as quasi-normal approximation (e.g., [44, 88, 89]), and it is known to have several drawbacks; positivity of the energy spectrum is not ensured, and it fails to recover the Kolmogolov spectrum in the inertial range of turbulence.

Nevertheless, the advantage of this treatment, similar to our closure approximation, is that the power spectrum can be computed numerically by solving the evolution equations. This forward treatment seems quite efficient to bring out the non-perturbative effects incorporated into the formalism, and it has a wide applicability to include various physical effects. Recently, the formalism has been extended to deal with the effect of massive neutrinos [90].

Valageas (2007) : The method proposed by Ref. [42] is based on the path-integral formalism. Starting from the action for the cosmological fluid equation (2.2), which describes the statistical properties of the vector field $\Phi_{a}$, the large-N expansions as a technique of quantum field theory have been applied to derive the governing equations for power spectrum and propagator. In Ref. [42], two kinds of expansions have been presented, leading to the two different non-perturbative schemes, i.e., steepest descent method and 2PI effective action method. Although both methods consistently reproduce the standard PT at the one-loop level, the latter includes the non-purturbative contributions which are not properly taken into account by the former method. Thus, the 2PI effective action method is expected to provide a better result. It is interesting to note that despite the field-theoretical derivation, the resultant governing equations for the 2PI effective action method turn out to be mathematically equivalent to those obtained from the closure approximation [43]. Hence, the diagrammatic representation of this formalism is exactly the same as shown in Fig. 2 ,

Matsubara (2008a) : Finally, we briefly mention the treatment proposed by Ref. [35]. This is the Lagrangianbased approach, and we begin by writing down the exact expressions for matter power spectrum in terms of the displacement vectors. The resultant expression is in the exponential form, and the purterbative expansions are then applied for the explicit calculation of the ensemble average. While a naive expansion of the displacement vectors, together with the solutions of Lagrangian PT, merely reproduces the (standard) Eulerian PT results, Ref. [35] has applied a partial expansion, and some of the terms have been kept in the exponential form. This can be interpreted as the partial resummation of a class of the infinite diagrams. The resultant expression for power spectrum is quite similar to the one-loop result of standard PT, but slightly differs from it in the sense that there appears the exponential prefactor. As a consequence, the prediction reasonably recovers the damping behavior of the BAOs seen in the N-body simulations, and it also explains the smearing effect on the baryon acoustic peak in the two-point correlation function.

One noticiable point of this method is that it is rather straightforward to generalize the calculations in real space to those in redshift space, since the displacement vectors in redshift space can be simply given by a linear mapping from those in real space. Further, the computational cost is less expensive compared to the other analytic methods. Although the validity range of this method is restricted to a narrow range of the low- $k$ modes, it would be very powerful for a fast compuation of the two-point correlation function.

\section{APPENDIX C: CONVERGENCE OF DIFFERENT COMPUTATIONAL METHODS FOR TWO-POINT CORRELATION FUNCTION}

In this paper, the grid-based calculation with FFT has been used for computing the two-point correlation functions from N-body data. Here, we compare it with other computational methods and check their convergences.

Fig. [15] shows the two-point correlation functions measured at $z=0.5$ from the single realization of wmap5 simulations. Upper-left panel shows the results from the direct pair-counting. For each particle, we randomly select pairs, which are accumulated for each bin of separations, allowing for oversampling. The estimated values of two-point correlation function are then plotted for different number of samples $N_{\text {sample }}: N_{\text {sample }}=640 \mathrm{k}, 2,560 \mathrm{k}, 10,240 \mathrm{k}$ and 40,960k. The resultant total number of pairs, $N_{\text {pair }}$, indicated in the panel is given by $N_{\text {pair }}=N_{\text {sample }} \times N_{\text {particle }}$, with $N_{\text {particle }}=512^{3}$ being the total number of particles. Note that the actual number of pairs that enters into the plotted range is less than $N_{\text {pair. }}$. On the other hand, upper-right panel shows the results from the grid-based pair-counting introduced by Barriga \& Gaztañaga (2002) 91] (see also Ref. 72]). In this method, we first construct the density field on a grid of $N_{\text {grid }}$ cells, and then estimate the correlation function through the pair count on grids:

$$
\widehat{\xi}\left(\left|\boldsymbol{r}_{i j}\right|\right)=\frac{1}{N_{\text {pair }}\left(\left|\boldsymbol{r}_{i j}\right|\right)} \sum_{i j} \delta\left(\boldsymbol{r}_{i}\right) \delta\left(\boldsymbol{r}_{j}\right)
$$



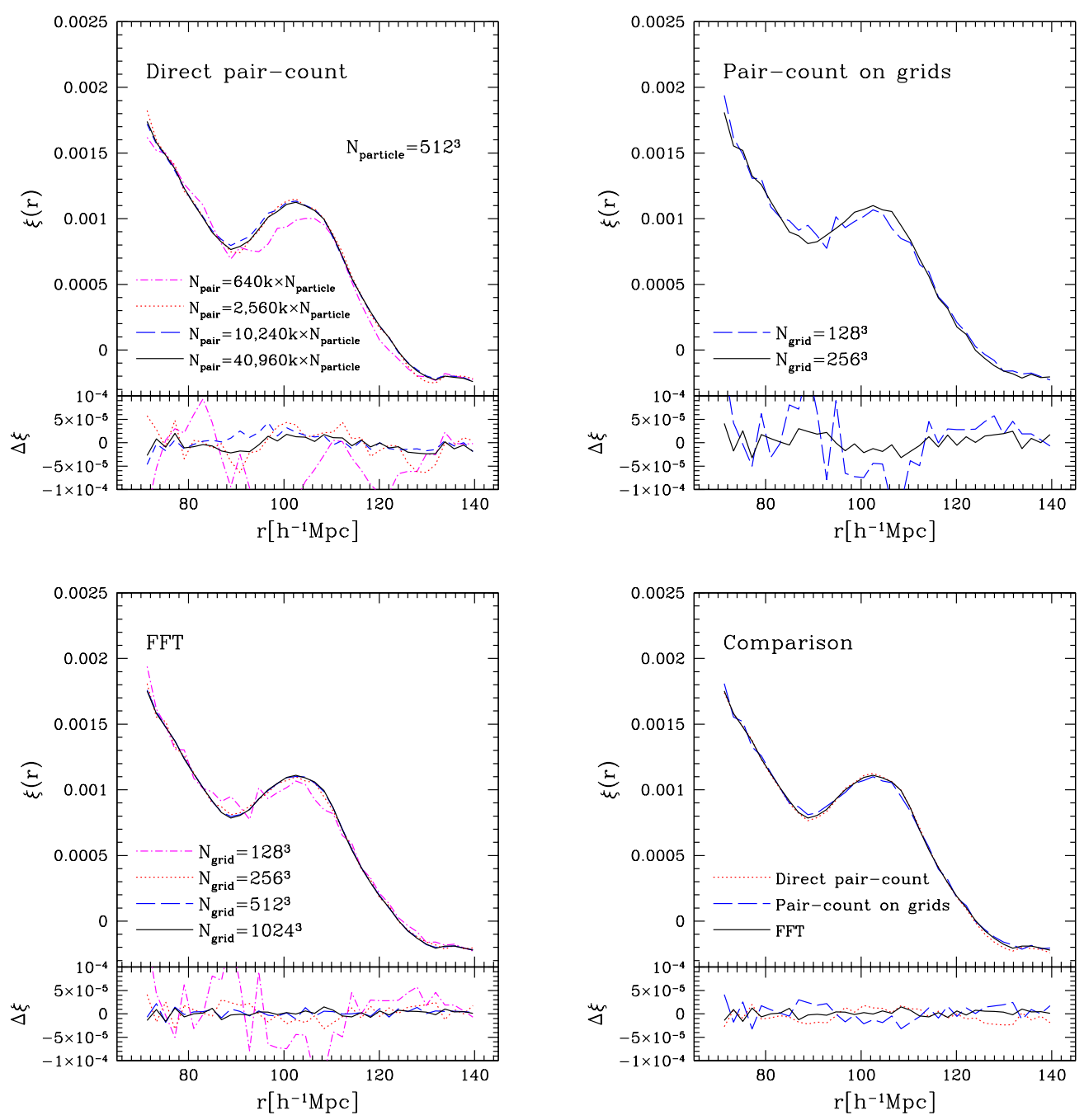

FIG. 15: Comparison between different computational methods for two-point correlation function.

Compared to the direct pair-counting, this method is computationally efficient when we store the list of neighbor particles which contribute to a given bin of separation. We plot the results adopting the two different number of cells, $N_{\text {grid }}=128^{3}$ and $256^{3}$. In lower-left panel, the grid-based calculation with FFT (see Eq. (4.2)) is used to compute the two-point correlation function, with different numbers of cells, $N_{\text {grid }}=128^{3}, 256^{3}, 512^{3}$ and $1,024^{3}$. Note that we adopt $N_{\text {grid }}=1,024^{3}$ in the analysis presented in Sec. IV Finally, in lower-right panel, the results for three different methods with the largest number of pairs or grids are collected and compared with each other.

To check the convergence, we further evaluate the residuals from the mean values, $\Delta \xi \equiv \widehat{\xi}-\bar{\xi}$, and plot the results in each panel of Fig. 15. Here, the mean values $\bar{\xi}$ are estimated from the ensemble average over the three different results using the largest number of pairs or grids. As increasing the numbers $N_{\text {pair }}$ or $N_{\text {grid }}$, the results for three different methods all approach the mean values $\bar{\xi}$, and a few percent-level agreement is achieved over the range of our interest (except for the vicinity of zero-crossing point, $\xi \approx 0$ ). It is interesting to note that residuals obtained from the grid-based pair-count and FFT methods almost coincide with each other and the differences are hard to distinguish, indicating that both methods are equivalent even in the practical situation. These experiments suggest that the grid-based calculation with FFT is a reliable estimation method comparable to the other methods. It should be emphasized that the method using FFT is much more efficient than other pair-count methods. For example, using 8 cores of $3 \mathrm{GHz}$ processors, the direct pair-counting with $N_{\text {pair }}=10,240 \mathrm{k} \times N_{\text {particle }}$ takes about two weeks to get the results shown in Fig. 15. The grid-based pair-counting is computationally less expensive than the direct pair-counting, but it still needs time-consuming calculations, especially for a large number of grids. By contrast, the method using 
FFT only requires few minutes even with $N_{\text {grid }}=1,024^{3}$. This can be achieved by a single-node calculation.

[1] D. N. Spergel et al. (WMAP), Astrophys. J. Suppl. 148, 175 (2003), astro-ph/0302209.

[2] D. N. Spergel et al. (WMAP), Astrophys. J. Suppl. 170, 377 (2007), astro-ph/0603449.

[3] E. Komatsu et al. (WMAP), Astrophys. J. Suppl. 180, 330 (2009), 0803.0547.

[4] M. Tegmark et al. (SDSS), Phys. Rev. D69, 103501 (2004), astro-ph/0310723.

[5] M. Tegmark et al. (SDSS), Phys. Rev. D74, 123507 (2006), astro-ph/0608632.

[6] S. Perlmutter et al. (Supernova Cosmology Project), Astrophys. J. 517, 565 (1999), astro-ph/9812133.

[7] A. G. Riess et al. (Supernova Search Team), Astron. J. 116, 1009 (1998), astro-ph/9805201.

[8] S. Nojiri and S. D. Odintsov, ECONF C0602061, 06 (2006), hep-th/0601213.

[9] R. Durrer and R. Maartens, Gen. Rel. Grav. 40, 301 (2008), 0711.0077.

[10] R. Durrer and R. Maartens (2008), 0811.4132.

[11] K. Koyama, Gen. Rel. Grav. 40, 421 (2008), 0706.1557.

[12] G. R. Dvali, G. Gabadadze, and M. Porrati, Phys. Lett. B485, 208 (2000), hep-th/0005016.

[13] W. Hu and I. Sawicki, Phys. Rev. D76, 064004 (2007), 0705.1158.

[14] A. A. Starobinsky, JETP Lett. 86, 157 (2007), 0706.2041.

[15] H.-J. Seo and D. J. Eisenstein, Astrophys. J. 598, 720 (2003), astro-ph/0307460.

[16] C. Blake and K. Glazebrook, Astrophys. J. 594, 665 (2003), astro-ph/0301632.

[17] D. J. Eisenstein et al. (SDSS), Astrophys. J. 633, 560 (2005), astro-ph/0501171.

[18] G. Huetsi, Astron. Astrophys. 449, 891 (2006), astro-ph/0512201.

[19] S. Cole et al. (The 2dFGRS), Mon. Not. Roy. Astron. Soc. 362, 505 (2005), astro-ph/0501174.

[20] W. J. Percival et al., Astrophys. J. 657, 51 (2007), astro-ph/0608635.

[21] W. J. Percival et al., Mon. Not. Roy. Astron. Soc. 381, 1053 (2007), 0705.3323.

[22] W. Hu and N. Sugiyama, Astrophys. J. 471, 542 (1996), astro-ph/9510117.

[23] D. J. Eisenstein and W. Hu, Astrophys. J. 496, 605 (1998), astro-ph/9709112.

[24] A. Meiksin, M. J. White, and J. A. Peacock (1998), astro-ph/9812214.

[25] H.-J. Seo and D. J. Eisenstein, Astrophys. J. 633, 575 (2005), astro-ph/0507338.

[26] A. J. Albrecht et al. (2006), astro-ph/0609591.

[27] J. A. Peacock et al. (2006), astro-ph/0610906.

[28] B. A. Bassett, R. C. Nichol, and D. J. Eisenstein (WFMOS) (2005), astro-ph/0510272.

[29] D. Schlegel, M. White, and D. Eisenstein (with input from the SDSS-III) (2009), 0902.4680.

[30] G. J. Hill et al. (2008), 0806.0183.

[31] X. Wang et al. (2008), 0809.3002.

[32] M. Crocce and R. Scoccimarro, Phys. Rev. D73, 063519 (2006), astro-ph/0509418.

[33] M. Crocce and R. Scoccimarro, Phys. Rev. D73, 063520 (2006), astro-ph/0509419.

[34] M. Crocce and R. Scoccimarro, Phys. Rev. D77, 023533 (2008), 0704.2783.

[35] T. Matsubara, Phys. Rev. D77, 063530 (2008), 0711.2521.

[36] T. Matsubara, Phys. Rev. D78, 083519 (2008), 0807.1733.

[37] P. McDonald, Phys. Rev. D75, 043514 (2007), astro-ph/0606028.

[38] K. Izumi and J. Soda, Phys. Rev. D76, 083517 (2007), 0706.1604.

[39] M. Pietroni, JCAP 0810, 036 (2008), 0806.0971.

[40] S. Matarrese and M. Pietroni, JCAP 0706, 026 (2007), astro-ph/0703563.

[41] P. Valageas, Astron. Astrophys. 421, 23 (2004), astro-ph/0307008.

[42] P. Valageas, Astron. Astrophys. 465, 725 (2007), astro-ph/0611849.

[43] A. Taruya and T. Hiramatsu, Astrophys.J. 674, 617 (2008), 0708.1367.

[44] D. Leslie, Developments in the Theory of Turbulence (Clarendon Press, Oxford, 1973).

[45] T. Nishimichi et al., Publ. Astron. Soc. Jap. 61, 321 (2009), 0810.0813.

[46] J. Carlson, M. White, and N. Padmanabhan (2009), 0905.0479.

[47] F. Bernardeau, S. Colombi, E. Gaztanaga, and R. Scoccimarro, Phys. Rept. 367, 1 (2002), astro-ph/0112551.

[48] T. Hiramatsu and A. Taruya (2009), 0902.3772.

[49] K. Koyama, A. Taruya, and T. Hiramatsu (2009), 0902.0618.

[50] R. E. Smith et al. (The Virgo Consortium), Mon. Not. Roy. Astron. Soc. 341, 1311 (2003), astro-ph/0207664.

[51] T. Hahn, Comput. Phys. Commun. 168, 78 (2005), hep-ph/0404043.

[52] V. Springel, Mon. Not. Roy. Astron. Soc. 364, 1105 (2005), astro-ph/0505010.

[53] M. Crocce, S. Pueblas, and R. Scoccimarro, Mon. Not. Roy. Astron. Soc. 373, 369 (2006), astro-ph/0606505.

[54] A. Lewis, A. Challinor, and A. Lasenby, Astrophys. J. 538, 473 (2000), astro-ph/9911177.

[55] R. Takahashi et al., Mon. Not. Roy. Astron. Soc. 389, 1675 (2008), 0802.1808.

[56] D. J. Eisenstein, H.-j. Seo, and . White, Martin J., Astrophys. J. 664, 660 (2007), astro-ph/0604361.

[57] R. E. Smith, R. Scoccimarro, and R. K. Sheth, Phys. Rev. D77, 043525 (2008), astro-ph/0703620.

[58] A. J. S. Hamilton (1997), astro-ph/9708102. 
[59] N. Kaiser, Mon. Not. Roy. Astron. Soc. 227, 1 (1987).

[60] J. A. Peacock and S. J. Dodds, Mon. Not. Roy. Astron. Soc. 267, 1020 (1994), astro-ph/9311057.

[61] S. Cole, K. B. Fisher, and D. H. Weinberg, Mon. Not. Roy. Astron. Soc. 267, 785 (1994), astro-ph/9308003.

[62] C. Park, M. S. Vogeley, M. J. Geller, and J. P. Huchra, Astrophys. J. 431, 569 (1994).

[63] W. E. Ballinger, J. A. Peacock, and A. F. Heavens, Mon. Not. Roy. Astron. Soc. 282, 877 (1996), astro-ph/9605017.

[64] R. Scoccimarro, Phys. Rev. D70, 083007 (2004), astro-ph/0407214.

[65] W. J. Percival and M. White, Mon. Not. Roy. Astron. Soc. 393, 297 (2009), 0808.0003.

[66] J. R. Shaw and A. Lewis, Phys. Rev. D78, 103512 (2008), 0808.1724.

[67] A. Meiksin and M. J. White, Mon. Not. Roy. Astron. Soc. 308, 1179 (1999), astro-ph/9812129.

[68] R. Scoccimarro, M. Zaldarriaga, and L. Hui, Astrophys. J. 527, 1 (1999), astro-ph/9901099.

[69] R. Takahashi et al., Astrophys. J. p. in press (2009), 0902.0371.

[70] J. D. Cohn, New Astron. 11, 226 (2006), astro-ph/0503285.

[71] G. M. Bernstein, Astrophys. J. 424, 569 (1994).

[72] A. G. Sanchez, C. M. Baugh, and R. Angulo, Mon. Not. Roy. Astron. Soc. 390, 1470 (2008), 0804.0233.

[73] A. F. Heavens, S. Matarrese, and L. Verde, Mon. Not. Roy. Astron. Soc. 301, 797 (1998), astro-ph/9808016.

[74] D. Jeong and E. Komatsu, Astrophys. J. 691, 569 (2009), 0805.2632.

[75] R. E. Smith, R. Scoccimarro, and R. K. Sheth, Phys. Rev. D75, 063512 (2007), astro-ph/0609547.

[76] P. McDonald, Phys. Rev. D74, 103512 (2006), astro-ph/0609413.

[77] P. McDonald and A. Roy (2009), 0902.0991.

[78] A. Taruya, Astrophys. J. 537, 37 (1999), astro-ph/9909124.

[79] K. Heitmann, D. Higdon, C. Nakhleh, and S. Habib, Astrophys. J. 646, L1 (2006), astro-ph/0606154.

[80] K. Heitmann et al. (2009), 0902.0429.

[81] S. Habib, K. Heitmann, D. Higdon, C. Nakhleh, and B. Williams, Phys. Rev. D76, 083503 (2007), astro-ph/0702348.

[82] M. H. Goroff, B. Grinstein, S. J. Rey, and M. B. Wise, Astrophys. J. 311, 6 (1986).

[83] T. Nishimichi et al., Publ. Astron. Soc. Jap. 59, 1049 (2007), 0705.1589.

[84] J. N. Fry, Astrophys. J. 421, 21 (1994).

[85] Y. Suto and M. Sasaki, Phys. Rev. Lett. 66, 264 (1991).

[86] N. Makino, M. Sasaki, and Y. Suto, Phys. Rev. D46, 585 (1992).

[87] R. Scoccimarro and J. Frieman, Astrophys. J. 473, 620 (1996), astro-ph/9602070.

[88] I. Proudman and W. H. Reid, Phil. Trans. Roy. Soc. London. Ser. A247, 163189 (1954).

[89] T. Tatsumi, Proc. Roy. Soc. London. Ser. A239, 16 (1957).

[90] J. Lesgourgues, S. Matarrese, M. Pietroni, and A. Riotto (2009), 0901.4550.

[91] J. Barriga and E. Gaztanaga, Mon. Not. Roy. Astron. Soc. 333, 443 (2002), astro-ph/0112278. 